University of Tennessee Health Science Center UTHSC Digital Commons

\title{
Tamoxifen: Mechanisms of Resistance
}

Cyrus McCoy Adams

University of Tennessee Health Science Center

Follow this and additional works at: https://dc.uthsc.edu/dissertations

Part of the Medical Pharmacology Commons, Neoplasms Commons, and the Pharmacy and Pharmaceutical Sciences Commons

\section{Recommended Citation}

Adams, Cyrus McCoy , "Tamoxifen: Mechanisms of Resistance" (2010). Theses and Dissertations (ETD). Paper 4. http://dx.doi.org/10.21007/etd.cghs.2010.0004.

This Thesis is brought to you for free and open access by the College of Graduate Health Sciences at UTHSC Digital Commons. It has been accepted for inclusion in Theses and Dissertations (ETD) by an authorized administrator of UTHSC Digital Commons. For more information, please contact jwelch30@uthsc.edu. 


\title{
Tamoxifen: Mechanisms of Resistance
}

\begin{abstract}
The role of estrogen in breast cancer has been recognized for decades. The selective estrogen receptor modulator tamoxifen was the first targeted therapy for the treatment of breast cancer. It was also the first drug approved by the FDA for the reduction of breast cancer risk. While tamoxifen has extended the lives of countless patients with breast cancer, resistance to tamoxifen remains a significant clinical problem. Work over the last two decades has greatly enhanced our understanding of the molecular mechanisms by which breast cancer cells may become resistant to tamoxifen treatment. Here I review our current understanding of the tamoxifen's mechanism of altering estrogen signaling along with the current experimental and clinical work investigating the mechanisms by which breast cancer cells develop resistance. Elucidation of the molecular mechanisms underlying tamoxifen resistance will allow improvement of treatment by potentially enhancing the effects of tamoxifen while reducing the incidence of resistance.
\end{abstract}

Document Type

Thesis

Degree Name

Master of Science (MS)

Program

Pharmacology

Research Advisor

Dale P. Suttle, Ph.D., B.S.

Keywords

breast cancer; resistance; selective estrogen receptor modulator; tamoxifen

Subject Categories

Medical Pharmacology | Medicine and Health Sciences | Neoplasms | Pharmacy and Pharmaceutical Sciences 


\title{
Tamoxifen: Mechanisms of Resistance
}

\author{
A Thesis \\ Presented for \\ The Graduate Studies Council \\ The University of Tennessee \\ Health Science Center
}

In Partial Fulfillment

Of the Requirements for the Degree

Master of Science

From The University of Tennessee

\author{
By \\ Cyrus McCoy Adams \\ May 2010
}


Copyright (C) 2010 by Cyrus McCoy Adams

All rights reserved 


\section{ACKNOWLEDGEMENTS}

I would like to express my gratitude to everyone who has assisted me in my pursuit of a Master of Science in Pharmacology. I would like to specifically convey my gratitude to my research advisor Dr. Dale P. Suttle, and to my committee members, Drs. Leonard Lothstein and Trevor W. Sweatman for their advice and experience. Lastly, I must thank my family and friends for their love and support. 


\begin{abstract}
The role of estrogen in breast cancer has been recognized for decades. The selective estrogen receptor modulator tamoxifen was the first targeted therapy for the treatment of breast cancer. It was also the first drug approved by the FDA for the reduction of breast cancer risk. While tamoxifen has extended the lives of countless patients with breast cancer, resistance to tamoxifen remains a significant clinical problem. Work over the last two decades has greatly enhanced our understanding of the molecular mechanisms by which breast cancer cells may become resistant to tamoxifen treatment. Here I review our current understanding of the tamoxifen's mechanism of altering estrogen signaling along with the current experimental and clinical work investigating the mechanisms by which breast cancer cells develop resistance. Elucidation of the molecular mechanisms underlying tamoxifen resistance will allow improvement of treatment by potentially enhancing the effects of tamoxifen while reducing the incidence of resistance.
\end{abstract}




\section{TABLE OF CONTENTS}

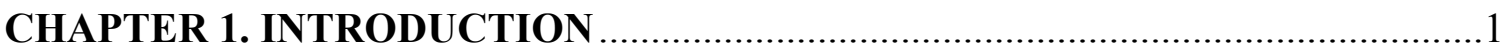

1.1. Endocrine Therapy for Breast Cancer......................................................... 1

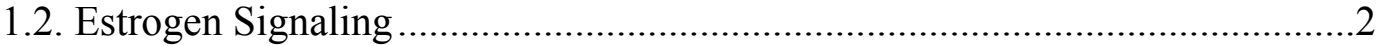

1.2.1. Estrogen Receptor Subtypes ................................................... 2

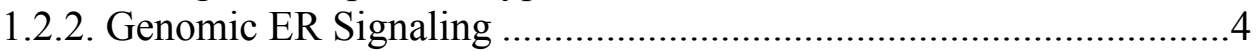

1.2.2.1. Classical Genomic Mechanism.......................................4

1.2.2.2. Non-Classical Genomic Mechanism................................6

1.2.3. Non-Genomic Mechanism ...........................................................6

1.3. Therapeutic Approaches to Target Estrogen Activity ..................................

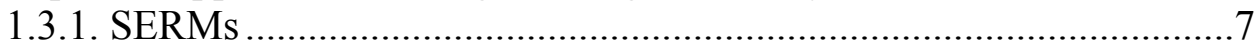

1.3.2. Aromatase Inhibitors........................................................... 9

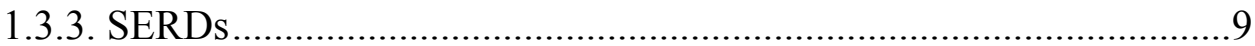

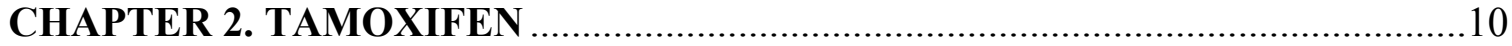

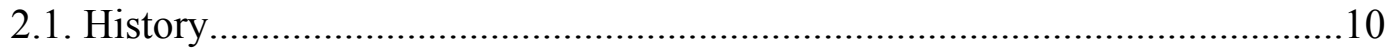

2.2. Mechanism of Action.............................................................................. 10

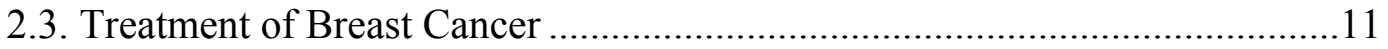

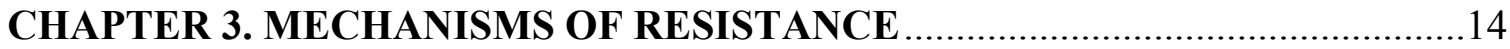

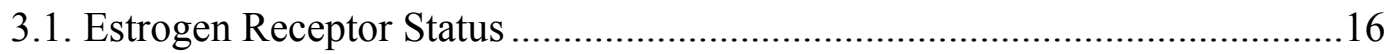

3.1.1. Loss of ER $\alpha$ Expression.........................................................16

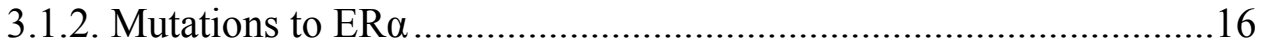

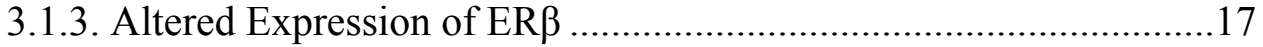

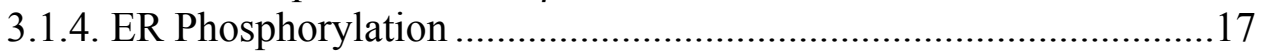

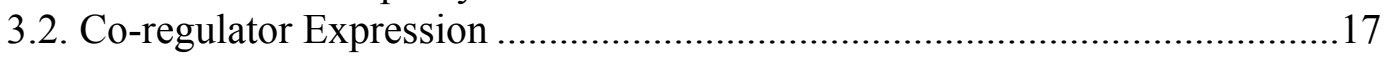

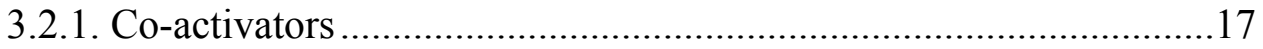

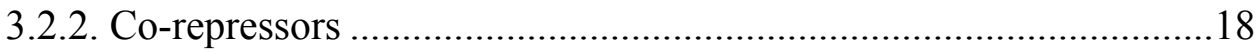

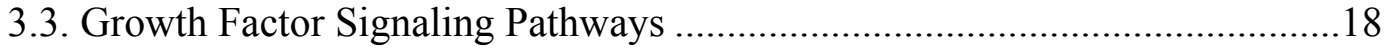

3.3.1. EGFR and HER2 2..................................................................19

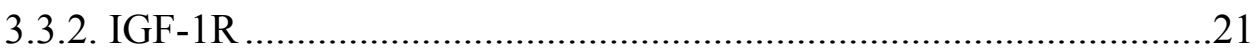

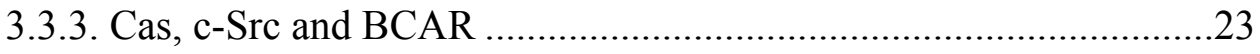

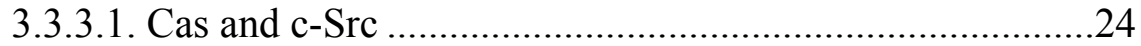

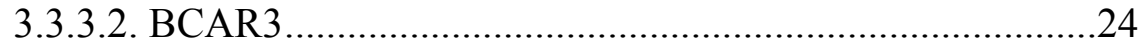

3.3.4. PI3K and AKT .......................................................................24

3.3.5. Stress-Activated Protein Kinase/c-Jun Kinase Pathway ..................25

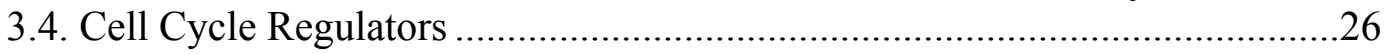

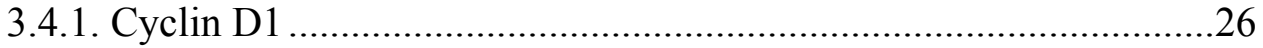

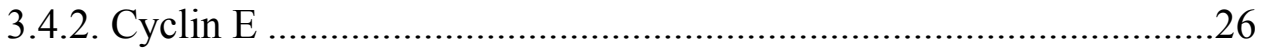

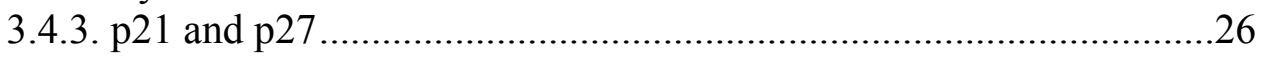

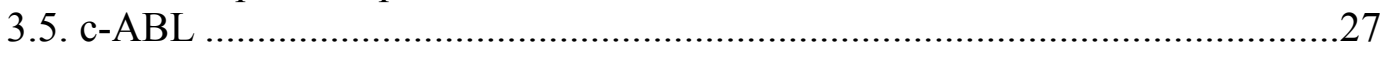

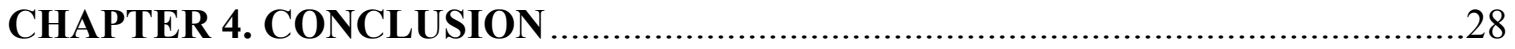




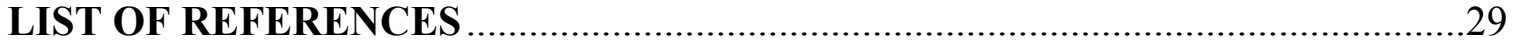

VITA 


\section{LIST OF FIGURES}

Figure 1-1 Human ER $\alpha$ and ER $\beta$ nuclear receptors .............................................

Figure 1-2 Estrogen receptor signaling pathways ............................................

Figure 1-3 Chemical structures of $17 \beta$-Estradiol and the three FDA-approved

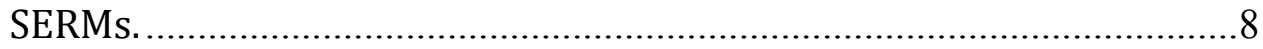

Figure 2-1 The potential actions of co-regulators at the ER .....................................12

Figure 3-1 The metabolism of tamoxifen ............................................................... 15

Figure 3-2 ER crosstalk with growth factor receptor pathways .............................20

Figure 3-3 Autocrine signaling loop counteracting the anti-proliferative effect of

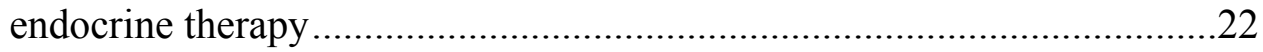




\section{CHAPTER 1. INTRODUCTION}

The American Cancer Society estimates that in 2009, 192,370 new cases of invasive breast cancer and 62,280 additional cases of in situ breast cancer were diagnosed among women, as well as approximately 40,170 deaths resulting from breast cancer [1]. Treatments for breast cancer include surgery, radiation therapy, and systemic therapy. Surgery is aimed at removing the cancer from the breast and assessing the stage of disease, where as radiation therapy may be used to destroy the cancer cells remaining after surgery. Systemic therapy may be neoadjuvant, often used to reduce the size of the tumor before surgery, or adjuvant to kill remaining undetected cancer cells. Systemic therapy includes biologic therapy, chemotherapy, and hormone therapy. Endocrine therapy is primarily aimed at interfering with estrogen signaling because of the important role estrogen plays in many breast cancers. Tamoxifen was the first targeted therapy for breast cancer as well as the first preventative, however resistance remains a significant issue. A clearer understanding of the molecular mechanisms by which resistance develops may prevent its occurrence and optimize treatment of patients with breast cancer.

\subsection{Endocrine Therapy for Breast Cancer}

The relationship between hormone therapy and breast cancer was first acknowledged over a century ago when surgeon Sir George Beatson preformed an oophorectomy on a patient with advanced breast cancer and observed a dramatic response [2]. He had no knowledge of the estrogen receptor, but simply noted that removing the ovaries, the major source of estrogen, could benefit women at the inoperable stage of disease. The estrogen receptor (ER) was reported in 1973 [3] and the association between the risk of breast cancer and elevated estrogen has been consistently found in many subsequent studies [4]. Estrogen stimulates the proliferation of breast epithelial cells, and has been implicated in the pathogenesis of breast cancer [5].

A meta-analysis of 51 epidemiologic studies enrolling 161,116 women investigated the relation between risk of breast cancer and use of hormone replacement therapy and showed that breast cancer risk increased by $2.3 \%$ per year of hormone use compared with an increased risk of $2.8 \%$ per year of natural delay in the onset of menopause [6]. These results imply that hormone use increases the risk of developing breast cancer and support the role of estrogen in the pathogenesis of breast cancer. However, before the introduction of pharmacological agents, endocrine therapy for breast cancer was ablative to remove sources of estrogen by either surgery or radiotherapy [7].

Allen and Doisy first discovered the estrogenic hormones produced in the ovary [8]. The discovery of estrogen led to questions regarding the mechanism by which estrogens exert their tissue specificity and if there is a target to block estrogen action. Previously it was believed that estrogens exert their actions by participating in enzymatic processes. However, the identification of the estrogen receptor that mediates the diverse 
actions of estrogen without alteration of the hormone itself provided a mechanism to describe the specificity of action and opened the door to molecular targeting in the treatment and prevention of breast cancer [9]. Two thirds of breast cancers express the estrogen receptor, therefore, ER-targeted therapy is widely used in breast cancer to inhibit signaling through ER and disrupt breast cancer growth [10].

\subsection{Estrogen Signaling}

Estrogen and estrogen receptors are key regulators of breast epithelial proliferation, differentiation, and apoptosis [11]. Estrogens are cyclopentanophenanthrene compounds whose synthesis begins with cholesterol like other sex and adrenal hormones. The most potent and abundant estrogen in humans is $17 \beta$-estradiol (E2), but estrone and estriol are also present at lower levels [12]. Because of their steroid structure, estrogens can diffuse passively through the cytoplasmic and nuclear membranes in order to interact with its nuclear receptor. Estrogen plays a broad role in human physiology and has been implicated in the development or progression of numerous diseases, such as various types of cancer, osteoporosis, neurodegenerative and cardiovascular diseases, insulin resistance, lupus erythematosus, endometriosis, and obesity [13]. Two molecular forms of the estrogen receptor (ER) have been identified, and ER acts as a transcriptional factor and also interacts with other transcriptional factors and growth factor-dependent kinases.

\subsubsection{Estrogen Receptor Subtypes}

Estrogen mediates its effects via receptors, which serve as the basis for many therapeutic interventions. ER is a ligand-activated transcription factor. It belongs to a superfamily of nuclear receptors that includes other steroid hormone receptors. Jensen and Jacobsen first demonstrated the existence of this receptor that could bind $17 \beta$ estradiol in the late 1950s, and the first ER was cloned in 1986 [14]. It was believed this was the single receptor until Kuiper et al. cloned another ER in 1996 from a prostate cDNA library. Today, the two estrogen receptors are designated ER $\alpha$ and ER $\beta$. The two proteins are produced by two distinct genes on different chromosomes and display tissue specific expression, with overlapping distribution: "ER $\beta$ is more expressed in prostate, bone, ovaries (granulosa cells), lungs, and in various parts of the central and peripheral nervous system, while ER $\alpha$ is predominantly expressed in the pituitary gland, ovaries (thecal and interstitial cells), uterus, liver, kidneys, adrenals, and the mammary glands" [15]. It is important to note that both subtypes are expressed in the breast.

$\mathrm{ER} \alpha / \beta$ are proteins sharing common regions, designated $\mathrm{A} / \mathrm{B}, \mathrm{C}, \mathrm{D}, \mathrm{E}$, and $\mathrm{F}$. These regions participate in the formation of independent but interacting functional domains [12]. Figure 1-1 shows the well-defined domain organization of the two receptors, which show a high degree of similarity at the amino acid level, as well as the degree of homology in the specific domains. The A/B domain includes the activation function 1 (AF1), which is the "region responsible for the constitutive and ligand- 


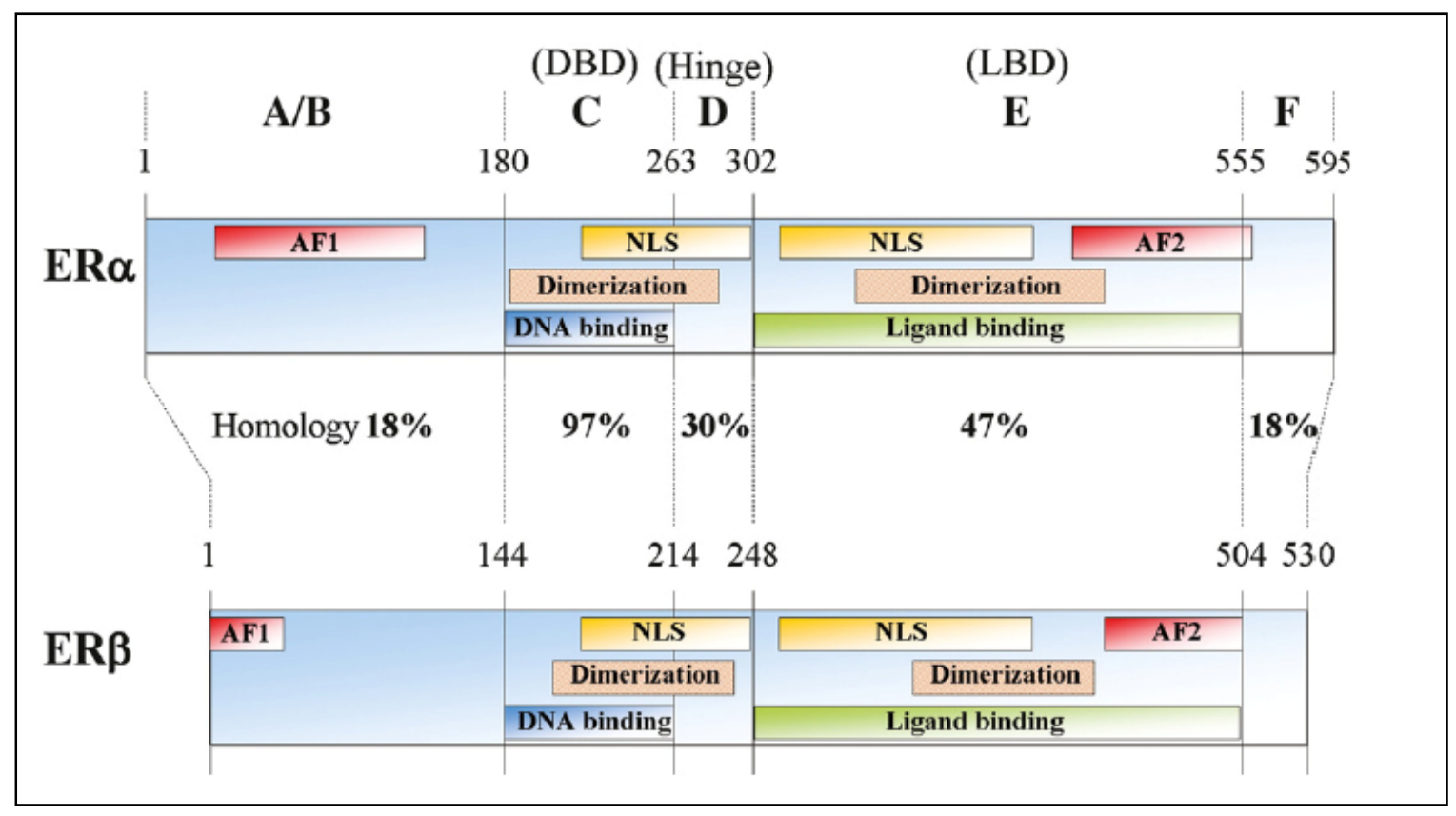

Figure 1-1. Human ER $\alpha$ and ERß nuclear receptors. A schematic representation of the structure of human ER $\alpha$ and ER $\beta$ is depicted. The numbers outside each box refer to the amino-acid number, and the percentage of amino-acid homology for each domain is also shown. The location of each domain on each receptor is designated.

Reprinted with permission. Zilli, M., et al., Molecular mechanisms of endocrine resistance and their implication in the therapy of breast cancer. Biochim Biophys Acta, 2009. 1795(1): p. 62-81 [15]. 
independent transcriptional activity of ER." The C domain contains the DNA-binding domain (DBD) and is also important for receptor dimerization. The D domain is a flexible hinge with a nuclear localization signal, and the $\mathrm{E}$ domain is the ligand-binding domain (LBD) and "harbors a second nuclear localization signal and the activation function 2 (AF2) responsible for the ligand-dependent activation of ER." The F domain is not essential and plays a complex modulatory role on both activities [15].

Soon after the identification of ER $\beta$, it was demonstrated that several compounds show receptor selectivity in binding and/or efficacy, and studies have since shown that ER $\alpha$ and ER $\beta$ have distinct biological activities. ER $\alpha$ is the stronger transcriptional activator of the two ER isoforms, at physiological concentrations of estradiol, coexpression of ER $\beta$ results in suppression of both the efficacy and the potency of hormone-stimulated responses, and therefore the relative expression levels of the two isoforms are an important determinant of cellular sensitivity to estrogens [16].

Many compounds could no longer be classified simply as "estrogenic" after the identification of the tissue specific actions of estrogens and the discovery of the role of a second estrogen receptor. ER $\beta$ ligands do not stimulate uterine growth or induce the progesterone receptor in the uterus, and ER $\alpha$ ligands do not inhibit prostatic growth, and the term "selective estrogen receptor modulator" (SERM) was introduced to provide a more generic description of the activity of an ER ligand [14].

\subsubsection{Genomic ER Signaling}

Estrogen influences the physiology of many target tissues and the long-term effects of estrogen are mediated predominantly via ERs functioning as DNA-binding transcription factors. ERs regulate gene expression by both direct (classical) and indirect (non-classical) binding of an ER to DNA (Figure 1-2). These pathways are referred to as genomic or "nuclear-initiated steroid signaling (NISS)" pathways [15]. Tissue-specific responses are a result of the regulation of different genes by ER.

\subsubsection{Classical Genomic Mechanism}

ERs predominantly exist as monomers bound to heat shock proteins in the cell nucleus. However, estrogen diffuses into the nucleus and binds to ER leading to conformational changes, phosphorylation and disassociation from the chaperon proteins and finally dimerization [17]. The ER dimmer may then bind to an estrogen response element (ERE) upstream of estrogen-dependent genes in the regulatory regions of target genes. The consensus palindromic ERE was initially described based on the estrogenresponsive sequence from estrogen-responsive genes from the Xenopus laevis A2 promoter: 5'-GGTCAnnnTGACC-3', but the human genome possesses imperfect palindromic sequences in their promoter with 3,4 , and 5 bp spacers [12]. 


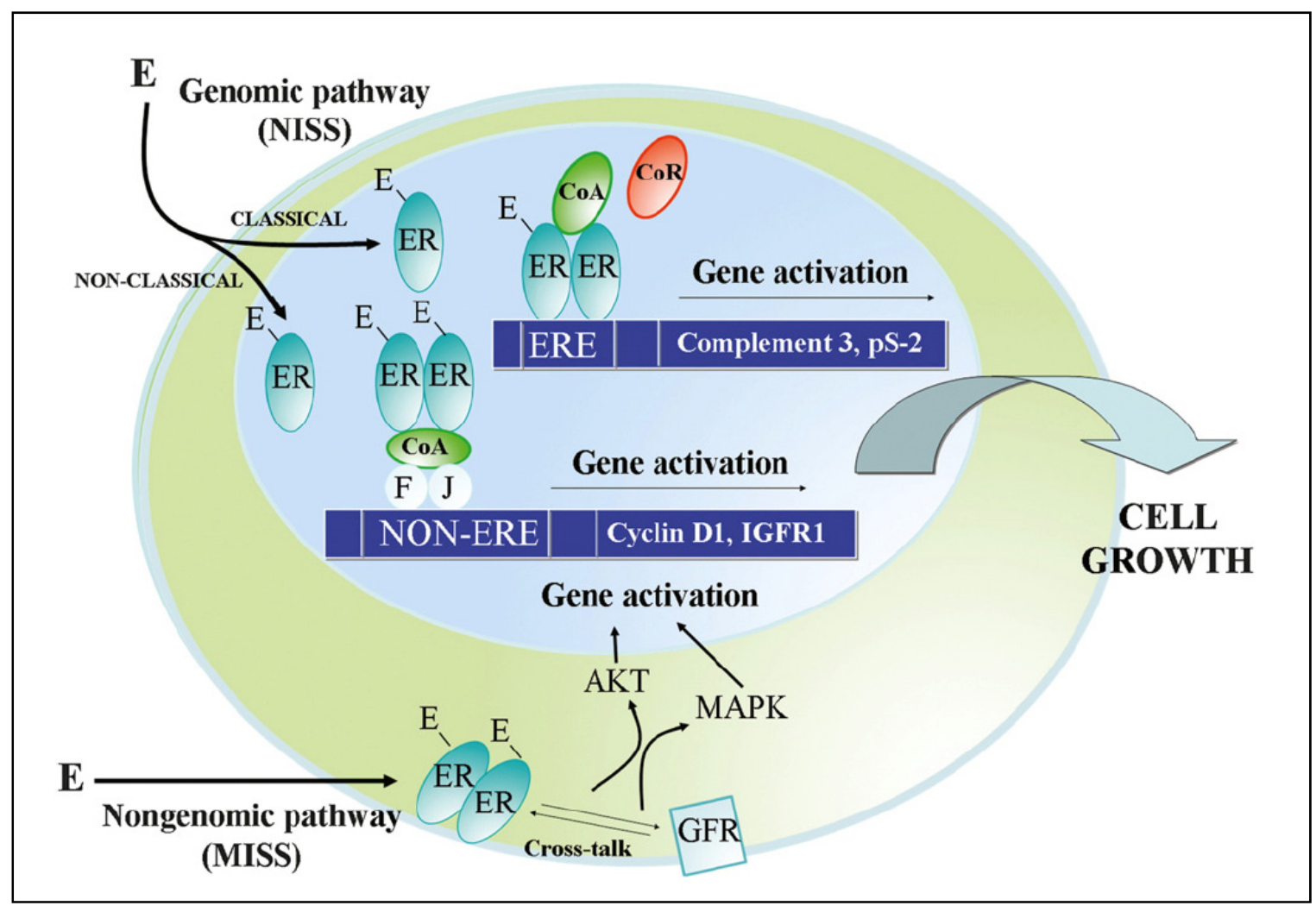

Figure 1-2. Estrogen receptor signaling pathways. This illustrates the genomic or "nuclear-initiated steroid signaling" (NISS) pathways and the non-genomic or "membrane-initiated steroid signaling" (MISS) pathway and the mechanisms by which they regulate cell growth.

Reprinted with permission. Zilli, M., et al., Molecular mechanisms of endocrine resistance and their implication in the therapy of breast cancer. Biochim Biophys Acta, 2009. 1795(1): p. 62-81 [15]. 


\subsubsection{Non-Classical Genomic Mechanism}

ERs may also influence gene expression without directly binding to DNA by interacting with other transcription factors. Such factors include Fos/Jun activating protein (AP-1) complex, cyclic AMP-response elements (CREs), and Sp1 sites and, this is the mechanism by which estrogen regulates cyclin D1 and IGFR1 [15].

Via both the classical and non-classical mechanisms, estrogen results in a general up-regulation of genes regulating cell proliferation and survival and the down-regulation of genes with anti-proliferative or pro-apoptotic activity and the final result growth stimulation and apoptosis suppression [18]. Up-regulation is mediated by the two transactivation domains mentioned above: AF-1 (A/B domain) and AF-2 (E domain). AF-1 activity is regulated by phosphorylation and is hormone-independent, while AF-2 is in the ligand-binding domain and therefore hormone-dependent [17].

Estrogen-bound ER can directly or indirectly induce gene transcription only if AF1 and/or AF2 are activated, and either domain may be dominant depending on the cellular environment or the specific promoter type, but generally the two act in synergy [15]. Ligand binding results in a conformational change of ER that uncovers AF-2 and that exposes a binding surface for further modulation by co-regulatory proteins. The binding of co-activators enhances the transcriptional activity by forming large complexes that may recruit histone-acetyltransferase (HAT) leading to chromatin decondensation, while co-repressors reduce transcription by recruiting histone-deacetylase (HDAC) to the promoter site resulting in chromatin condensation [15]. The co-regulatory proteins recruited are affected by the ER conformation which is dependent on the ligand and the specific ERE sequence to which the ER is bound [19].

Unlike AF-2, AF-1 is ligand independent and activated by phosphorylation at serine residues subsequent to activation of kinase pathways triggered by growth factor receptors [15]. Epidermal growth factor receptor (EGFR), insulin-like growth factor-1 receptor (IGFR), and human epidermal growth factor receptor type 2 (HER2) have been implicated in this mechanism [17]. The mechanism by which estrogen affects AF-1 is not well understood at this point. It is important to note that in the absence of estrogen, minimal phosphorylation is responsible for constitutive AF-1 activity and that growth factor signaling pathways can directly activate ER and subsequent transcription. The growth factor signaling will be explained further in Section 3.2.2. as a potential mechanism of resistance to endocrine therapy.

\subsubsection{Non-Genomic Mechanism}

The membrane-initiated steroid signaling (MISS) pathway is a rapid (in seconds to minutes) non-genomic mechanism of action, mediated by membrane-associated ER. This occurs via a population of ERs that exists within caveolar rafts and other domains in the plasma membrane. ER is associated with caveolin-1 and a variety of proximal signaling molecules, including G proteins, Src and Ras, and B-Raf. Activation of these 
ERs results in signal cascades from $G$ protein activation. Similarly to many other $G$ protein coupled receptors, G protein activation by ER leads to the cell-specific stimulation of phospholipase C, protein kinase C, ERK, or phosphatidylinositol 3-kinase and nitric-oxide synthase [20]. The activation of these kinases has the ability to activate growth factors and cytoplasmic kinases, which may in turn phosphorylate ER and its coregulators. This suggests that the non-genomic and genomic activities are complementary and potentially synergistic [15]. Non-genomic activity is highly dependent on ligand and is regulated by co-regulatory proteins. The activity is also strongly dependent on the signal transduction pathways operating within the cell [15].

\subsection{Therapeutic Approaches to Target Estrogen Activity}

The search for pharmacologic means of antagonizing the biological effects of estrogen as a therapeutic strategy for women with breast cancer has been ongoing ever since the link between human breast cancer and estrogen emerged. A strategy to antagonize estrogen in an attempt to treat/prevent breast cancer may have a severe impact on a patient's health by interfering with the significant physiological roles of estrogen in reproductive tissues, bone, the cardiovascular system and its role in cognitive function and behavior [21].

"Hormone-receptor (HR) status is an important factor used to determine the prognosis and treatment of breast cancer" [22]. HR-positive breast cancer may be ERpositive, Progesterone-Receptor-positive or both, and typically responds to treatments that specifically block or interfere with the function of estrogen or progesterone. Sensitive tumors are now identified by testing for specific receptors, the degree of positivity scored, and taken into account when determining a course of therapy. Of 5,993 mammary carcinomas examined by immunohistochemistry by Nadji et al., 75\% were positive for ER and 55\% of all tumors reacted positively for PR. All PR-positive tumors were also positive for ER, and endocrine therapy is indicated as part of adjuvant treatment for these tumors [23]. There are now several effective options for the treatment of hormone-receptor positive breast cancer and include selective ER modulators (SERMs), aromatase inhibitors, and selective ER down-regulators (SERDs).

\subsubsection{SERMs}

SERMs exert selective agonist or antagonist effects on various estrogen sensitive tissues. They are a chemically diverse group of compounds that lack the steroid structure of estrogens, and although the primary structure of SERMs differ strikingly from that of estrogens (Figure 1-3), they have a tertiary structure that permits their binding to the ligand-binding domain of the ER [24]. Several of these drugs have been available for decades, but their tissue-specificity in humans has only recently been acknowledged. The current clinically available SERMs are Tamoxifen, Toremifene, and Raloxifene, and they differ slightly in their actions and side effects. The specific mechanism of actions of SERMs with focus on Tamoxifen will be discussed in greater detail in Section 2. 


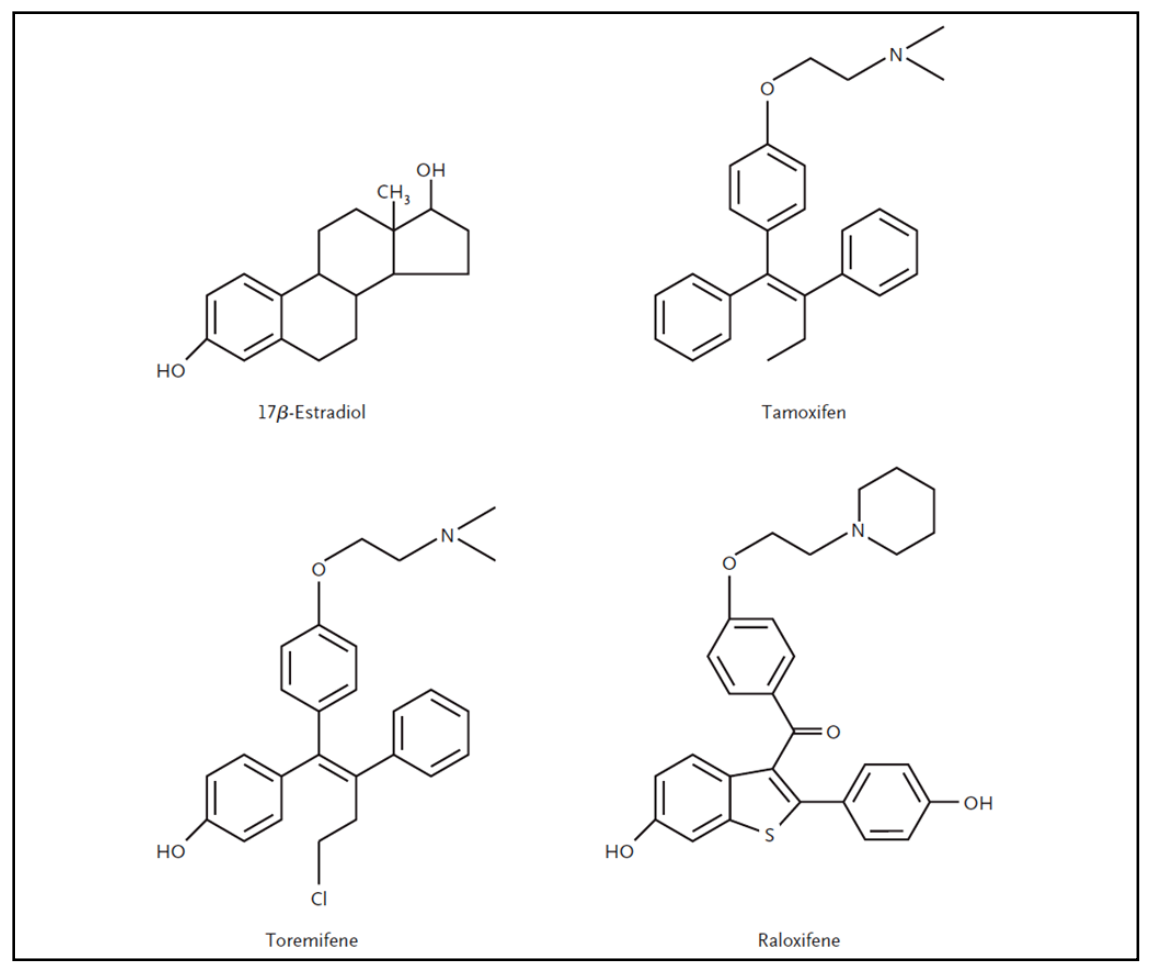

Figure 1-3. Chemical structures of 17 $\beta$-Estradiol and the three FDA-approved SERMs. 


\subsubsection{Aromatase Inhibitors}

Anti-aromatase agents inhibit the cytochrome P-450 component of the aromatase enzyme complex, which is responsible for the final step of estrogen biosynthesis. These drugs are classified into generations, and the third-generation agents are currently approved for use in the treatment of breast cancer. These are further divided into type I and type II: Type I inhibitors (exemestane) have a steroidal structure similar to androgens and inactivate the enzyme irreversibly by blocking the substrate-binding site, while Type II inhibitors (anastrozole and letrozole) are non-steroidal and their action is reversible [25].

\subsubsection{SERDs}

SERDs are also termed 'pure anti-estrogens,' and only fulvestrant is being used clinically. It is showing promising clinical activity in the treatment of advanced breast cancer. Fulvestrant has a higher affinity for the estrogen receptor (ER) compared to SERMs, but does not have any of the agonist activities [21]. Fulvestrant is a novel ER antagonist that binds to the receptor and prevents ER dimerization, leading to rapid degradation and loss of cellular ER expression [26]. 


\section{CHAPTER 2. TAMOXIFEN}

\subsection{History}

For more than 30 years, tamoxifen has been the gold standard for the endocrine treatment of ER-positive breast cancer, and it is estimated that more than 400,000 women are alive today as a result of tamoxifen therapy, and millions more have benefited from palliation and extended disease-free survival [27]. The discovery of non-steroidal antiestrogens by the pharmaceutical industry during the 1960s was initially an exciting prospect for 'morning after' anti-fertility agents. Unfortunately, unlike in the rat, the antiestrogens actually improved fertility by inducing ovulation in subfertile women [28]. The discovery of the ER in the 1970s and the relationship between estrogen and breast cancer provided a target in the treatment and prevention of breast cancer. At this point research into anti-estrogens previously abandoned as anti-fertility agents was revived.

In the laboratory, tamoxifen was demonstrated to prevent the induction of carcinogen-induced rat mammary tumors and was shown to have efficacy in the treatment of advanced breast cancer and became the first targeted therapy for breast cancer when it was approved in 1977 for the treatment of women with metastatic breast cancer [29]. Over time the widening use of tamoxifen for long-term treatment and the reduction in the risk of contralateral breast cancer in adjuvant trials led to the evaluation of its use as a preventative for breast cancer. In 1992, the National Surgical Adjuvant Breast and Bowel Project (NSABP) implemented a randomized clinical trial to evaluate tamoxifen's potential in the prevention of breast cancer in 13,800 high risk women and found that breast cancer incidence was reduced by 50\% in tamoxifen-treated women compared to placebo treated controls [30]. In 1999, the Food and Drug Administration approved tamoxifen for the reduction of breast cancer risk, and later, it was the first drug approved for the prevention of any cancer. The reinvention of tamoxifen from a failed "morning after pill" to the first targeted therapy for breast cancer and first chemopreventive provided the clinical research community with an invaluable new therapeutic tool to pioneer the strategy of long term anti-hormonal therapy and chemoprevention [31].

\subsection{Mechanism of Action}

The mechanisms of tissue selective, mixed agonist-antagonist action of tamoxifen and other SERMs are still being determined, but the unique actions may be explained by interactive mechanisms. The fundamental elements that factor into the tissue-selective actions are the differential estrogen-receptor expression in a given target tissue, the differential ER conformation on ligand binding, the precise sequence compositions of the EREs, and the differential expression and binding of co-regulator proteins to the estrogen receptor [24]. Because of their distinct biological activities the relative expression of ER $\alpha$ and ER $\beta$ in different tissues will affect the response to both estrogens and tamoxifen. 
As explained in 1.2.1, ER $\alpha$ and ER $\beta$ have distinct biological activities and ER $\beta$ can in fact suppress the actions of ER $\alpha$ by forming a heterodimer with it. Thus, the relative levels of expression of these two receptor isoforms will affect the cellular responsiveness to estrogens. Tamoxifen binds to both isoforms and affects the cellular responsiveness of each [32]. SERMs function as pure antagonists at ER $\beta$ on genes containing estrogen response elements but function as partial agonists when acting via $\mathrm{ER} \alpha[16]$.

Recently, it has been demonstrated via protein crystallography and surface probing techniques that binding of tamoxifen to ER results in a unique conformational change. When estrogen binds to the hydrophobic pocket of ER $\alpha$, the pocket is closed by helix 12 of the protein resulting in the activation of AF-2, but when tamoxifen binds to the pocket, it prevents reorientation of helix 12 [33]. The precise composition of the ERE's can also alter the structural conformation of the receptor.

Many co-regulator proteins bind to ER and modulate ER function. As ER regulates the rate of gene transcription through its association with co-regulators, the overall balance of expression levels of co-activators and co-repressors have been shown to be an important determinant of the tissue-specificity of SERMs (Figure 2-1). For example, tamoxifen recruits a co-activator complex to ER genes in endometrial cells where it acts as an agonist, but a co-repressor complex to the same gene in the breast where it acts as an antagonist [34]. Figure 2-1 is a schematic representing the role of coregulators in modulating receptor conformation and activity. When tamoxifen binds the receptor, the receptor adopts an intermediate partial conformation that is neither fully active nor fully inactive. In the intermediate conformation, the receptor is also subject to interactions with the relative intracellular concentrations of preferred co-activators or corepressors for its activity [32]. Depending on the unique receptor conformation induced by ligand binding, varying combinations of co-regulator proteins interact with the estrogen receptor and modulate its function in a variety of ways [24].

\subsection{Treatment of Breast Cancer}

Tamoxifen acts as an estrogen antagonist for the breast, and the tissue selective actions made it the gold standard treatment for ER-positive breast cancer. By acting as a weak ER agonist in the bone, tamoxifen reduces bone loss. Treatment also induces a beneficial serum lipid profile by decreasing LDL levels. However, tamoxifen therapy is also associated with an increase in the risk of endometrial cancer because of its actions as a partial agonist in the endometrium. Nevertheless, there have been numerous studies demonstrating efficacy for the treatment and prevention of ER-positive breast cancer. Tamoxifen is mainly cytostatic and acts by slowing the proliferation of breast cancer cells by inhibiting their progression from the G1 phase of the cell cycle, but it has also been shown to induce apoptosis in vitro in breast cancer cell lines and therefore may possess cytocidal properties in vivo as well [24].

Adjuvant tamoxifen therapy has been demonstrated to significantly reduce the 


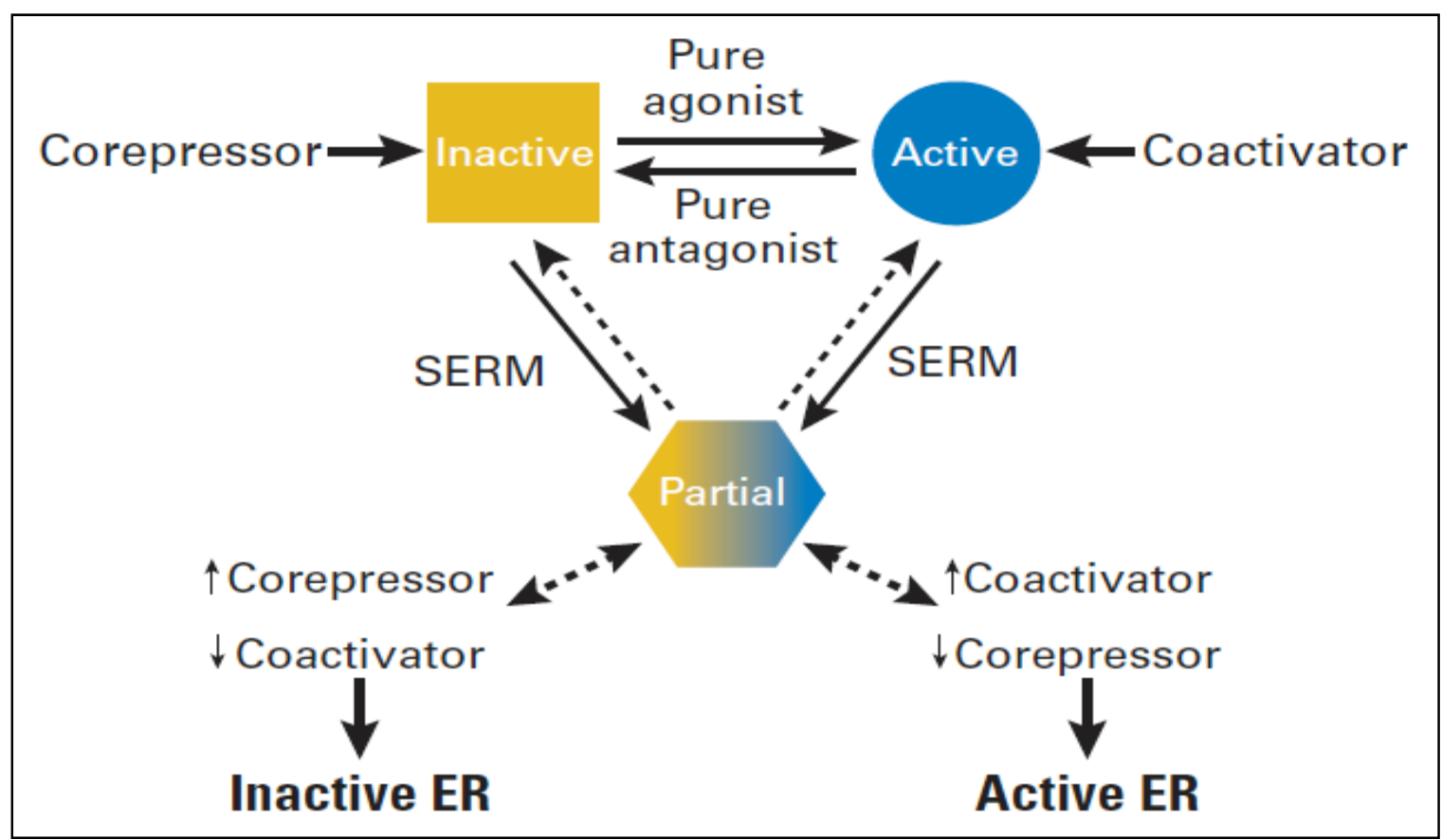

Figure 2-1. The potential actions of co-regulators at the ER. The schematic demonstrates the potential contributions of co-activators and co-repressors to the tissuespecific activities of a SERM.

Reprinted with permission. Jordan, V.C. and B.W. O'Malley, Selective estrogen-receptor modulators and antihormonal resistance in breast cancer. J Clin Oncol, 2007. 25(36): p. 5815-24 [32]. 
risk of recurrence and death from breast cancer. A review of 55 clinical trials of adjuvant therapy with 37,000 women with breast cancer examined tamoxifen versus no tamoxifen before recurrence. The proportional reduction in recurrence was $47 \%$ after 5 years of treatment with tamoxifen and the proportional reduction in mortality was $26 \%$ after 10 years [35]. The absolute improvements in 10-year survival were $10.9 \%$ in lymph nodepositive and $5.6 \%$ in lymph node-negative breast cancer, and women with ER-negative cancer had very limited benefit [24]. As discussed in Chapter 2.1, the widening use of long-term tamoxifen treatment led to its inclusion in randomized primary-prevention trials, and the NSABP trial demonstrated a $50 \%$ reduction in the risk of breast cancer in high risk patients when compared to placebo treated controls [30]. 


\section{CHAPTER 3. MECHANISMS OF RESISTANCE}

Despite the relative safety and significant anti-neoplastic and chemopreventive activities of tamoxifen, most initially responsive breast tumors develop resistance [33]. The development of resistant tumors remains a major obstacle in the struggle to treat and prevent breast cancer. Approximately 30\% of ER-positive breast cancers do not respond to tamoxifen treatment (de novo resistance), and of the tumors that initially respond to treatment, the majority develops resistance over time (acquired resistance), despite the continued expression of ER. Studies have shown that ER continues to regulate tumor development in most resistant cases and the majority are still responsive to fulvestrant and AIs, indicating that estrogen remains an important regulator of tumor growth [11]. Drug resistance to anti-hormonal therapy in breast cancer was originally viewed as the ER-negative cells overgrowing ER-positive cells whose growth had been arrested from tamoxifen treatment; however, this view is no longer accepted. It is unlikely that one single mechanism can explain hormonal resistance in all breast cancer patients, but recent work has provided details about the types of resistance and the mechanisms by which they may develop.

Three classes of resistance are acknowledged: metabolic resistance, intrinsic (or de novo) resistance, and acquired resistance [32]. Tamoxifen is a prodrug that is metabolically activated. Generated via CYP3A4/5 and CYP2D6 (Figure 3-1), 4hydroxytamoxifen and especially endoxifen are important active metabolites of tamoxifen that are necessary to achieve the maximal anti-estrogenic and antitumor effects of tamoxifen. These metabolites have been shown to posses a 100-fold greater affinity for ER and 30- to 100- fold greater potency in suppressing ER-dependent proliferation and gene expression [15]. Wide variations in both of these enzymes responsible for tamoxifen metabolism exist in the population and the clinical response to tamoxifen therapy is likely to depend on the cumulative effect of these metabolites [36]. The clinical relevance of these variants on patient outcomes in the treatment of breast cancer with tamoxifen is unclear and must be addressed in large populations and with prospective studies [37].

Intrinsic resistance is exemplified by tumors that fail to respond to initial tamoxifen therapy, while acquired resistance develops over the time course of tamoxifen therapy. The remainder of this review will focus on these types of resistance. Such mechanisms include modifications of ER status and alterations in the intracellular environment via changed expression of co-regulators and the influence of kinase and growth factor signaling.

Tamoxifen's effects are mediated via the ER, and therefore the degree of expression of ER is a strong predictor of response to tamoxifen treatment, and the status of ER expression is critical [17]. As explained previously, tamoxifen acts as an antagonist at $\mathrm{ER} \alpha$, but may act as an agonist to ER $\beta$. The loss of expression, mutation or change in activity of $E R \alpha$, as well as an alteration in the expression of $\operatorname{ER} \beta$ are potential mechanisms by which ER status may change, thus affecting tamoxifen's actions and resulting in the development of clinical resistance. 


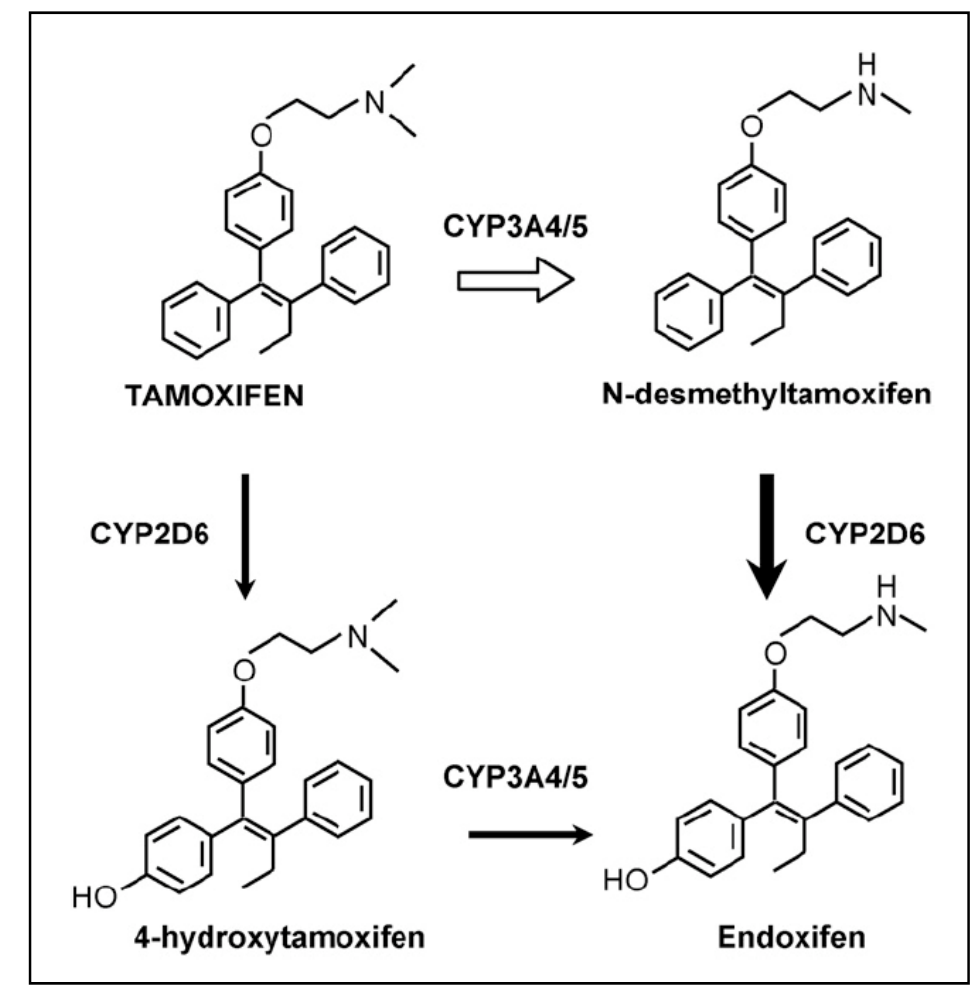

Figure 3-1. The metabolism of tamoxifen. Tamoxifen is metabolized via CYP3A4/5 and CYP2D6 to its active metabolites 4-hydroxytamoxifen and endoxifen.

Reprinted with permission. Jordan, V.C., New insights into the metabolism of tamoxifen and its role in the treatment and prevention of breast cancer. Steroids, 2007. 72(13): p. 829-42 [37]. 


\subsection{Estrogen Receptor Status}

\subsubsection{Loss of ER $\alpha$ Expression}

The expression of ER $\alpha$ has long been regarded as a strong predictor of clinical response to tamoxifen and any anti-estrogen therapy. Anti-estrogen therapies are only effective in cancers that are ER-positive and dependent on ER signaling for growth and proliferation. Loss of ER $\alpha$ may confer resistance to any anti-estrogen therapy, and the lack of ER expression is the primary mechanism of de novo or intrinsic resistance to tamoxifen [15]. The large majority of ER-negative breast cancer tumors demonstrate intrinsic resistance, but a small percentage of $E R \alpha$-negative tumors that are progesterone receptor (PgR) positive respond to anti-estrogen treatment. PgR is considered another important indicator of anti-estrogen response because studies show that roughly $70 \%$ of ER $\alpha$-positive/PgR-positive tumors effectively respond to tamoxifen, while only $34 \%$ of $\mathrm{ER} \alpha$-positive/PgR-negative tumors respond [11]. "The predictive power of PgR expression is likely related to the ability of estrogens to induce its expression. Thus, the presence of both ER and PgR may reflect the existence of an at least partially functional ER signaling pathway" [38].

Alterations of the ER $\alpha$ gene in the form of point mutations, deletions, or insertions are rare events that do not explain the ER-negative status of $20-30 \%$ of breast cancers and epigenetic mechanisms are now being investigated [39]. Work in cultured human breast cancer cells previously demonstrated that the absence of ER gene expression is associated with an increased capacity to methylate DNA, as well as extensive methylation of $\mathrm{CpG}$ islands of the ER gene. These results suggest that abnormal methylation may account for transcriptional inactivation [40]. Other work suggests that increased deacetylation of histones via HDAC may result in the loss of ER expression.

It was once thought that the loss of ER expression may be responsible for acquired resistance to tamoxifen, but only a relatively small percentage of tumors with acquired resistance do not express ER [41]. Many tamoxifen resistant patients will still respond to second line anti-estrogen treatment with fulvestrant or an aromatase inhibitor indicating a continued role of estrogen and the ER in the progression of the cancer.

\subsubsection{Mutations to ERa}

Several studies have demonstrated that mutations of ER can confer resistance to tamoxifen. A naturally occurring mutation of ER $\alpha$ has been identified that results in increased estrogen sensitivity and increased proliferation at lower estrogen concentrations [42]. However, it has been reported that only $1 \%$ of the primary breast cancers have point mutations in the ER gene [43]. Therefore such mutations of ER are unlikely to contribute significantly to the development of resistance to tamoxifen. These types of point mutations as well as deletions, duplications, insertions and alternative splicing events 
have the potential to regulate ER function and therefore should be considered, but resistance commonly develops in their absence.

\subsubsection{Altered Expression of ERß}

The data regarding the expression level of ER $\beta$ and tamoxifen resistance is conflicting. As discussed previously, ER $\beta$ has the ability to repress the activity of ER $\alpha$ [44]. Studies have reported low levels of ER $\beta$ to be predictive of tamoxifen resistance, while others have shown high levels of ER $\beta$ in resistant breast cancer cells [15]. Like $\mathrm{ER} \alpha, \mathrm{ER} \beta$ expression in breast cancer is associated with a better prognosis. However, the contrasting results of studies make a conclusion regarding the role of ER $\beta$ in tamoxifen resistance unfeasible at this point in time, and more work is necessary to establish whether ER $\beta$ expression is important in tamoxifen resistance.

\subsubsection{ER Phosphorylation}

ER is a target of serine, threonine and tyrosine phosphorylation, and ER phosphorylation is a key element of non-genomic actions in response to estrogen, but has also been shown to affect genomic transcription mediated events [11]. Introduction of mutations at these sties of phosphorylation often results in enhancement of ER transcriptional activity. The kinases that mediate these phosphorylations include Src, PAK1, PKA, cyclin A, AKT and ERK1/2. Many of the potential mechanisms discussed below alter the phosphorylation and thereby the activity of ER possibly contributing to the resistant phenotype.

\subsection{Co-regulator Expression}

Co-regulators play an important role in mediating the transcriptional activity of ER. Because many of these may be present at rate-limiting levels in the nucleus, changes in their level of expression and/or activity can lead to alterations of ER signaling [15]. In particular, over expression of co-activators and down regulation of co-repressors may contribute to tamoxifen resistance. "An imbalance in the expression of co-activator and co-repressor genes may impair tamoxifen activity either by abolishing the anti-estrogenic effect or by switching the effect from anti-estrogenic to estrogenic," but few studies have been studied the role of co-regulators in tamoxifen resistance [45].

\subsubsection{Co-activators}

In vitro studies have demonstrated that increased co-activator expression can enhance the agonist activity of tamoxifen. AIB1(also called SRC-3, RAC3, ACTR, and $\mathrm{p} / \mathrm{CIP}$ ) is an ER co-activator thought to be important in breast cancer. AIB1 is phosphorylated and activated by mitogen-activated protein kinases (MAPKs), and high 
levels of activated AIB1 may potentially reduce the antagonist effects of tamoxifen, especially in tumors that also over-express the HER-2 receptor, a member of the epidermal growth factor (EGF) receptor family that activates MAPKs [46]. A1B1 is overexpressed in over $50 \%$ of breast tumors [47], and enhances the agonist effect of tamoxifen in vitro [48].

A retrospective study investigated the hypothesis that tumors with a relatively high abundance of AIB1 are less responsive to tamoxifen therapy in 187 patients having received adjuvant tamoxifen and 119 patients with no adjuvant treatment. High AIB1 expression in patients not receiving adjuvant tamoxifen therapy was associated with better prognosis and longer disease free survival. In contrast, for patients who did receive tamoxifen therapy, high AIB1 expression was associated with worse disease free survival, which may be indicative of tamoxifen resistance [46]. Patients with tumors expressing high levels of both AIB1 and HER-2 had worse outcomes with tamoxifen therapy than all other patients combined suggesting a cross-talk between ER and growth factor signaling pathways (discussed in Section 3.3.). This work supports the hypothesis that increased expression or activation of ER co-activators could enhance the agonist activity of tamoxifen and potentially contribute to resistance.

\subsubsection{Co-repressors}

The co-repressor NCoR has been shown to interact with ER when bound to $4 \mathrm{OH}-$ tamoxifen and acts as part of larger protein complex with histone deacetylase activity that promotes chromatin condensation and leads to target-gene silencing [45]. NCoR binds avidly to ER in the presence of tamoxifen, but when NCoR activity is blocked with an antibody in MCF-7 human breast cancer cells, tamoxifen instead acts as an agonist [49]. MCF7 cells were also implanted into ovarectomized, athymic nude mice and the animals were subsequently treated with tamoxifen. NCoR levels declined in many of the tumors that acquired resistance to tamoxifen, relative to tumors with continued response. This data demonstrates that a decrease in expression of NCoR can result in a change in tamoxifen activity and potentially contribute to resistance.

Girault et al. analyzed the mRNA expression (RT-qPCR) of NCoR in a welldefined series of ER-positive breast cancer patients who exclusively received adjuvant tamoxifen therapy after surgery. They demonstrated that low expression of NCoR is associated with significantly shorter relapse-free survival in tamoxifen treated patients [50]. Both this work in vivo and the work in vitro demonstrate a mechanism by which down regulation or decreased activity of the co-repressor NCoR can contribute to the development of resistance to tamoxifen.

\subsection{Growth Factor Signaling Pathways}

ER signaling does not exist in isolation within the intracellular environment, and many other elements, most notably those comprising growth factor transduction cascades, 
can influence or be influenced by ER signaling [51]. As previously described, in addition to the genomic actions, ER may initiate rapid cellular signaling via direct interaction with components of growth factor signaling pathways. Activation of ER via the non-genomic pathway outside the nucleus results in phosphorylation, and activation of surface tyrosine kinase receptors (such IGF-IR, EGFR, and HER2) and also with cellular kinases and adaptor molecules (such as c-Src and PI3K) [10]. Many of these interactions lead to the activation of key downstream signaling kinases such as MAPK and AKT, which also have the potential to phosphorylate and thereby activate ER itself or its co-activator proteins (Figure 3-2) [10].

Alterations in these signaling cascades have the ability to alter the therapeutic responses to anti-estrogenic drugs such as tamoxifen and potentially lead to the development of resistance. The inappropriate activation of growth factor signaling cascades can potentially occur through an enhanced supply of growth factor ligands, via up-regulation or through increased activation of the receptors or their downstream signaling cascades [52]. Such changes can potentially stimulate growth and proliferation of breast cancer cells independently of ER or via cross-talk with ER, and thereby contribute to resistance.

\subsubsection{EGFR and HER2}

Epidermal growth factor receptor (EGFR) and Human epidermal growth factor receptor 2 (HER2) are transmembrane receptor tyrosine kinases that signal via MAPK, ERK1/2, PI3K, and AKT to stimulate proliferation, differentiation, and cell survival. Dysregulation and over-expression of EGFR can lead to transformation of cells in vitro and tumor formation in nude mice [53]. "Increased EGFR, its cognate growth factor ligands (EGF, TGF- $\alpha$, amphiregulin), or hyper-activation of its signaling elements have invariably been associated with anti-hormonal resistance, aggressive clinicopathology, disease metastasis, and poor prognosis" [54]. In vitro transfection studies indicate a causal role of EGF receptor expression in breast cancer progression to hormone independence [55]. Other investigators have demonstrated that acquisition of resistance in ER-positive breast cancer cell lines following long term treatment with tamoxifen often coincides with over-expression of EGFR and activation of downstream molecules [11].

ER is a regulator of the cell-cycle, and estrogen-activated ER results in a negative feedback to control cell growth by down-regulating genes involved in proliferation, such as EGFR and HER2 [15]. In the presence of estrogen, ER-positive breast cancer cells suppress EGFR expression, but tamoxifen, by antagonizing ER, may result in increased expression and activation of these signaling pathways. EGFR activates MAPK and AKT to stimulate proliferation and cell survival, and these signaling kinases may also phosphorylate and thereby activate ER or its co-activator proteins resulting in increased expression of ER genes [10]. Two ER regulated genes of note are the EGFR ligands amphiregulin and TGF $\alpha$. Through this mechanism, tamoxifen may enhance the transcription of these ligands creating an autocrine loop that maintains cell growth and 


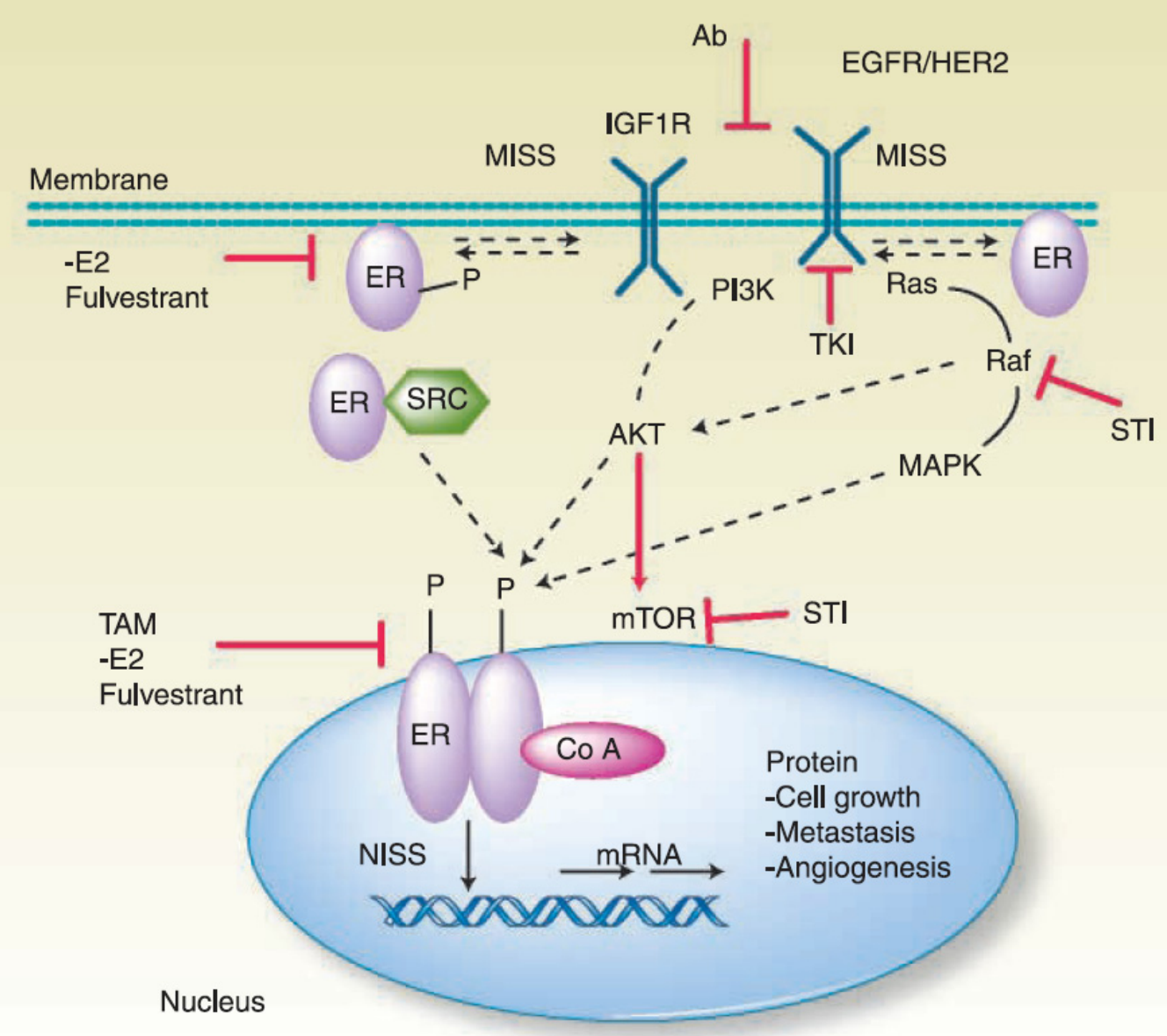

Figure 3-2. ER crosstalk with growth factor receptor pathways. This signaling diagram illustrates the potential crosstalk between ER and many of the growth factor pathways discussed that potentially contribute to tamoxifen resistance in breast cancer.

Reprinted with permission. Massarweh, S. and R. Schiff, Unraveling the mechanisms of endocrine resistance in breast cancer: new therapeutic opportunities. Clin Cancer Res, 2007. 13(7): p. 1950-4 [10]. 
proliferation despite tamoxifen treatment [56]. An example of an autocrine signaling loop resulting in cell growth despite anti-hormone treatment is depicted in Figure 3-3.

There is substantial evidence supporting the up-regulation of EGFR as a mechanism for the enhanced cross talk with ER; however, another group has demonstrated another potential mechanism to enhance the interaction between EGFR and ER [57]. Fan et al. demonstrated that long-term exposure of hormone-sensitive MCF-7 breast cancer cells to tamoxifen causes cytoplasmic translocation of ER $\alpha$. They report that the elevation in the levels of extra-nuclear ER $\alpha$ increases the opportunity of ER to associate with factors that facilitate activation of the MAPK pathway. The enhanced interaction between ER $\alpha$ and EGFR is associated with an increased ability of EGF, estrogen and tamoxifen to stimulate MAPK, and tamoxifen elicited rapid phosphorylation of MAPK in resistant cells in contrast to its antagonistic activity in control cells [57]. Their results further support that enhanced non-genomic function of ER via cooperation with the EGFR pathway represents a mechanism responsible for acquired tamoxifen resistance.

HER2 has also been implicated in de novo resistance to tamoxifen. Breast cancer patients whose ER-positive tumors express high levels of the co-activator AIB1 and HER2 treated with tamoxifen experience substantially more recurrences than those with ER-positive tumors that have lower expression of one or both proteins [46]. Tamoxifen acts as an antagonist in parental MCF-7 cells, which express high AIB1 but low HER2 expression, and tamoxifen behaves as an agonist in MCF-7 cells expressing high levels of both HER2 and AIB1 [58]. This agonist activity on gene expression and cell proliferation resulted in tumor growth stimulation by tamoxifen demonstrating de novo resistance. In this intracellular environment, tamoxifen-bound ER recruits the co-activator rather than co-repressors, resulting in agonist activity [15]. Both the clinical and in vitro data indicate that both HER2 and AIB1 are necessary for this resistant phenotype. This mechanism of resistance is also a function of enhanced cross-talk as the enhanced signaling of HER2 results in the phosphorylation and activation of both ER and the co-activator AIB1. The agonist effect of tamoxifen in the resistant cells is reversed by administration of gefitnib, a tyrosine kinase inhibitor [58]. Gefitnib restores the recruitment of co-repressor complexes by tamoxifen-bound ER resulting in a return of the antagonistic effects.

\subsubsection{IGF-1R}

The role of IGF signaling in tamoxifen resistance is much less clear than that of EGFR and HER2, but evidence for a role of IGF-1R is emerging [11]. IGF-1R is also a member of the receptor tyrosine kinase family, and it is activated after ligand binding of insulin, IGF-I, or IGF-II resulting in the activation of downstream signaling pathways including AKT, MAPK and PI3K cascades [58]. As previously discussed these signaling pathways have been shown to influence cell survival and proliferation. IGF-IR is an ER-dependent gene and tamoxifen, which effectively inhibits classically ERdependent transcription, can effectively reduce its expression [59]. The data regarding the 


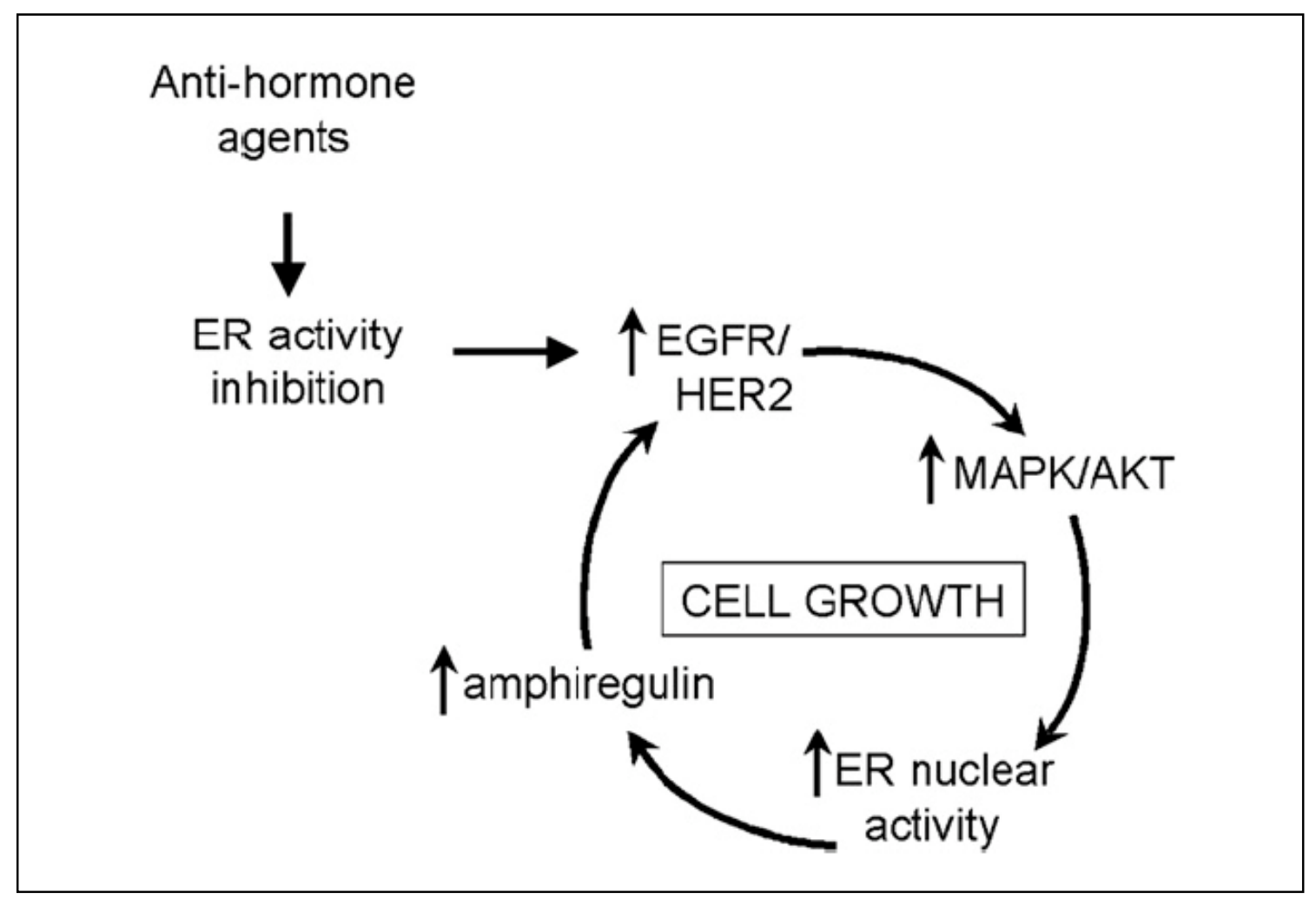

Figure 3-3. Autocrine signaling loop counteracting the anti-proliferative effect of endocrine therapy. Anti-estrogen treatment may result in an increase of EGFR/HER2 expression. These receptor tyrosine kinases activate the downstream MAPK/AKT signaling cascade, which phosphorylate and activate nuclear ER in the absence of ligand. Persistent ER activation leads to increased transcription of ER-sensitive genes, including amphiregulin and TGF, which also activate EGFR completing the autocrine proliferative loop. The net effect is a reduction or elimination of the anti-proliferative effect of endocrine therapy.

Reprinted with permission. Zilli, M., et al., Molecular mechanisms of endocrine resistance and their implication in the therapy of breast cancer. Biochim Biophys Acta, 2009. 1795(1): p. 62-81 [15]. 
levels of IGF-1R in tamoxifen-resistant breast cancer lines is mixed, however there is increasing evidence supporting synergistic interactions between IGF-1R, ER and EGFR. Estrogens result in an increase in expression of the IGF-IR and growth, while IGFs prime the activation of several kinases that are able to phosphorylate ER and initiate estrogenmediated gene expression [58].

One mechanism by which IGF-1-treated breast cancer cells may escape tamoxifen-induced apoptosis is via the IGF-mediated activation of AKT and subsequent phosphorylation of ER leading to the ligand-independent activation of ER [60]. Recent in vitro studies have aimed at elucidating the role of IGF-1R in breast cancer resistance. Unlike EGFR, several studies have reported that tamoxifen continues to suppress IGF-1R level even during the development of resistance. However, Massarweh et al. discovered that p-IGF-IR, although initially reduced in the tamoxifen-sensitive tumors, is increased at the time of resistance, suggesting that IGF-IR becomes activated at the time of acquired resistance and then augments important cell survival pathways [59]. It has also been shown that inhibition of IGF-1R (by an anti-IGF-1R antibody [61] or by an IGF-1R tyrosine kinase inhibitor [58]) reduces growth of tamoxifen-resistant MCF-7 breast cancer cells. An in vivo model also demonstrated that an IGF-1R monoclonal antibody enhances the anti-tumor activity of tamoxifen in estrogen receptor positive breast cancer xenografts [62].

Data also suggest that bi-directional crosstalk pathways between the epidermal growth factor family of receptors (erbB) and IGF-1R exists, and this interaction has been suggested to in part mediate the development of acquired resistance. In vitro work has shown that increased IGF-IR signaling interferes with the action of the HER-2 antibody trastuzumab in HER2 positive breast cancer cells [63]. Similarly, these breast cancer cells displayed enhanced cytotoxic effects when cultured with trastuzumab and an anti-IGF1 receptor antibody in combination [64]. Both activated EGFR and HER2 are also sufficient for conferring resistance to an IGF-1R inhibitor [65]. These data suggest that alterations to both the IGF-1R and erbB receptor pathways may occur in a reciprocating fashion leading to resistance [66]. The mechanism by which IGF may interact with EGFR and ER in breast cancer cells is becoming clearer; however, to date no conclusive clinical data has been reported regarding the clinical significance of IGF-IR's role in tamoxifen resistance.

\subsubsection{Cas, c-Src and BCAR}

Several cytoplasmic proteins whose functions are also coupled to growth factor receptors have been connected to tamoxifen resistance. In particular, BCAR and Cas are two implicated proteins that were discovered in a screening to identify proteins capable of inducing anti-estrogen resistance in vitro [11]. It has since been noted that patients with primary breast tumors expressing high levels of Cas protein are associated with more rapid disease recurrence and have a greater risk of (intrinsic) resistance to tamoxifen [67]. 


\subsubsection{Cas and c-Src}

High expression of Cas (p130Cas) is associated with resistance to tamoxifen in both tissue culture and in human tumors. Cas is a scaffold protein that assists in the formation of multi-protein complexes, by promoting protein-protein interactions [68]. It is an important aspect of signal transduction pathways that modulate cell motility, survival and proliferation. Riggins et al. demonstrated that Cas binds to and activates cSrc, a protein tyrosine kinase and results in phosphorylation of c-Src substrates, such as EGFR and signal transducer and activator of transcription (STAT) 5b. Inhibition EGFR and STAT5b abrogates the effect of Cas overexpression, supporting the significance of these c-Src substrates in the development of resistance [69]. Pharmacologic inhibition of c-Src in MCF-7 cells also enhances the inhibitory effects of tamoxifen on cell growth [70]. It is clear that Cas overexpression diminishes the anti-proliferative and proapoptotic effects of tamoxifen on breast cancer cells in vitro, and data suggests it accomplishes this via the activation of the Cas/c-Src/EGFR/STAT5 signaling pathway.

Whether these pathways are also clinically significant in resistance to tamoxifen has not yet been established; however, a large percentage of human breast tumors express high levels of both c-Src and Cas, as well as elevated c-Src kinase activity, and "cSrc/Cas complexes can be isolated from a majority of breast cancer cell lines" [11]. These data together suggest that tamoxifen resistance may be mediated in part through this mechanism involving c-Src and Cas.

\subsubsection{BCAR3}

Breast cancer anti-estrogen resistance 3 (BCAR3) was discovered in a genetic screening for genes associated with anti-estrogen resistance. It encodes a component of intracellular signal transduction and interacts directly with Cas. Transfer of the BCAR3 gene by cell fusion or transfection to breast cancer cells induces anti-estrogen resistance suggesting that up-regulation of BCAR3 (also knowns as AND-34) stimulates an alternative growth path independent of hormone, both in the presence and absence of anti-estrogens [71]. BCAR3 has since been shown to alter the activity, expression, and intracellular location of many proteins, including Cas, Ras and Rho family GTPases, and Pak1 [11]. Work by Felekkis et al. showed BCAR3 over-expression induced antiestrogen resistance in human breast cancer cell lines by activating PI3K, which induced Rac activation and finally resulted in anti-estrogen resistance [72]. While the data suggest that BCAR3 over-expression has the potential to result in resistance in cultured cells, the role of BCAR3 in the clinical setting of resistance has not yet been established.

\subsubsection{PI3K and AKT}

Downstream of growth factor receptors and adapter protein activation, signaling intermediates such as AKT play an important role in cell proliferation, survival, and endocrine resistance [11]. Receptor tyrosine kinases, such as the growth factor receptors 
EGFR and IGF-1R, activate PI3K, which in turn activates AKT, leading to substrate phosphorylation. The tumor suppressor PTEN antagonizes AKT and acts as a counter balance acting as a phosphatase. AKT activation promotes cell survival by inhibiting apoptosis and also plays a roll in regulation of the cell cycle. Over-expression of constitutively active AKT in MCF-7 breast cancer cell lines results in estrogen independence and resistance to tamoxifen [73], and activation of AKT is increased in tamoxifen-resistant MCF-7 breast cancer cells [74]. The exact mechanisms by which AKT may confer anti-estrogen resistance are unclear.

Phosphorylation of ER by AKT is one proposed mechanism demonstrated in vitro to result in decreased sensitivity to tamoxifen. Phosphorylation of Ser-167 by AKT results in increased ER binding to DNA and increased recruitment of the co-activator SRC3 potentially resulting in increased agonism [75]. In ER-positive breast cancer cell lines, PI3K-mediated AKT phosphorylation of ER $\alpha$ results in receptor activation in the absence of estrogen [60]. AKT has also been shown to enhance co-activator recruitment to ER and to potentiate ER $\beta$ transcriptional activity [60]. Evidence from in vitro studies supports a role of AKT-mediated phosphorylation of ER, but the clinical significance in tamoxifen resistance remains unclear.

AKT can also indirectly activate mTOR which also controls cell growth, survival, and apoptosis [76]. In response to activation by growth factor receptors, AKT phosphorylates and inactivates the tuberous sclerosis complex, which acts as a negative regulator of mTOR. Blockage of the PI3K/AKT signaling pathway by pharmacologic mTOR inhibition successfully restores the effectiveness of tamoxifen in MCF-7 breast cancer cells with constitutively active AKT [77]. While a definitive role of mTOR in resistance has not been determined, mTOR is considered an attractive therapeutic target for treating breast cancer [11].

\subsubsection{Stress-Activated Protein Kinase/c-Jun Kinase Pathway}

Jun NH2-terminal kinases (JNKs) and stress activated protein kinases (SAPKs), which are activated by cellular stresses, increase AP-1 transcriptional activity via phosphorylation. AP-1 is a transcription factor heterodimer composed of Jun and Fos family members, which binds to DNA at AP-1 response elements [78]. In vitro work has demonstrated that the development of tamoxifen resistance is accompanied by increased AP-1 DNA binding [79]. Johnston et al. examined a panel of 30 ER-positive primary human breast tumors with acquired tamoxifen resistance, and these tumors also displayed a significant increase in AP-1 DNA binding activity when compared to untreated control tumors [80]. In a tamoxifen-resistant xenograft model, increased phosphorylated c-Jun and JNK levels accompanied the increase in AP-1 dependent transcription following tamoxifen treatment, and the conversion to a resistant phenotype was associated with an increase in oxidative stress [81]. It is known that tamoxifen can induce intracellular oxidative stress, and this data supports the model in which tamoxifen-induced oxidative stress leads to activation of JNK and SAPK resulting in increased AP-1 activity and could contribute to resistance [17]. 


\subsection{Cell Cycle Regulators}

Cyclins, cyclin-dependent kinases (CDKs), and CDK inhibitors are the major regulators of cell cycle progression [11]. Estrogen accelerates the progression from $\mathrm{G}_{1}$ to $\mathrm{S}$ phase, while tamoxifen inhibits cell cycle progression by affecting these key cell cycle proteins [82]. These expression and activity of these proteins have been demonstrated to have the potential to significantly impact tamoxifen sensitivity and resistance.

\subsubsection{Cyclin D1}

Essential for early progression through the $\mathrm{G}_{1}$ phase, cyclin D1 both modulates CDK4/6-dependent phosphorylation of the tumor suppressor Rb and sequesters the CDK inhibitors p21 and p27 [11]. Cyclin D1 is a direct transcriptional target of estrogen signaling, and therefore tamoxifen reduces cyclin D1 expression [83]. In vitro sustained expression of cyclin D1 is evident in breast cancer cells during their acquisition of tamoxifen resistance [84], and down-regulation of cyclin D1 with siRNA restored the sensitivity of these cells to tamoxifen [85]. Cyclin D1can potentiate the transcriptional activity of the ER independently of estrogen and may not be inhibited by tamoxifen [86]. Clinical studies also support the role of cyclin D1 in tamoxifen resistance. Patients with cyclin D1 negative tumors show better relapse free survival on tamoxifen, and cyclin D1 expression is correlated with a poorer outcome of tamoxifen treatment [87]. Multiple clinical studies have demonstrated that breast cancer patients also show that cyclin D1 over-expression correlates with poor outcome on tamoxifen treatment $[88,89]$.

\subsubsection{Cyclin $\mathrm{E}$}

Cyclin D1 inhibits Rb early in $\mathrm{G}_{1}$ phase, and the transcription factor E2F strongly induces the expression of cyclin E, which associates with CDK2 to form an active complex that promotes entry into $\mathrm{S}$ phase [90]. Cyclin $\mathrm{E}$ and $\mathrm{CDK} 2$ activity is antagonized by the CDK inhibitors p21 and p27. The contribution of cyclin E to tamoxifen resistance is unclear, but over-expression of cyclin E in MCF-7 cells partially counteracts the growth arrest mediated by tamoxifen [91]. More studies are necessary to determine the mechanisms by which cyclin $\mathrm{E}$ is induced in breast cancer and its role in the induction of resistance.

\subsection{3. $\mathrm{p} 21$ and $\mathrm{p} 27$}

p21 and p27 are CDK inhibitors and are negative regulators of cell cycle progression. These proteins counteract the activities of cyclin D1 and cyclin E. In MCF7 cells, tamoxifen increase the expression of p21 and p27 during cell cycle arrest, but down-regulation of these by antisense inhibition prevents the growth inhibitory effects [92]. p27 induction in breast cancer cells by tamoxifen induces quiescence and 
insensitivity to growth stimulation by growth factors such as IGF-I and EGF [93]. Somatic deletion of the $\mathrm{p} 21$ gene in human breast cancer cells resulted in hyperphosphorylation of ER $\alpha$ causing an increased gene expression of ER regulated genes [94]. These studies demonstrate that p27 and p 21 are critical for the inhibitory effects of tamoxifen in inhibiting breast cancer cell growth.

c-Myc, a transcription factor whose expression is frequently altered in human breast cancer, also plays a role in regulating the activity of p21. Increased c-Myc expression can rescue the growth arrest mediated by anti-estrogen treatment by activating CDK2/Cyclin E complex [95]. In vitro over-expression of c-Myc reduces the expression of p21 in response to tamoxifen potentially mediating tamoxifen resistance [96]. They also demonstrate that $\mathrm{p} 21$ expression in anti-estrogen resistant cells is increased when treated with c-Myc siRNAs.

Clinical data also supports a role of these CDK inhibitors in response to tamoxifen treatment. In premenopausal women with early breast cancer, an increase in p27/KIP1 expression was able to predict better relapse free survival upon tamoxifen combination treatment [97]. A multivariate analysis of their data revealed decreased p27 expression to be correlated with poor outcome upon combination endocrine therapy. Localization of these CDK inhibitors has also been implicated in the development of resistance. Perex-Tenorio et al. showed that increased activity of the PI3K and MAPK pathway promotes $\mathrm{p} 21$ localization into cytoplasm through phosphorylation of residues within their nuclear localization sequences [98]. The significance of their in vitro data is supported by immunohistochemistry in frozen human tumors. They demonstrated that increased cytoplasmic localization of p21 is correlated with a poorer response to tamoxifen treatment in a cohort of 280 women.

\section{5. c-ABL}

c-ABL is a non-receptor tyrosine kinase with multiple functions in the regulation of cell migration, responses to oxidative stress and DNA damage, cell proliferation, and survival [99]. The oncogenic potential of c-ABL was first discovered in leukemias, but has recently been found it is not confined to hematopoietic malignancies [100]. Studies now indicate that c-ABL has a potential role in breast carcinogenesis. Zhao et al. demonstrated that c-ABL interacts with ER resulting in enhanced ER activity and together promote resistance to tamoxifen [100]. They report that c-ABL is required for maintaining ER transcriptional activity and that silencing c-ABL results in the sensitization of ER-positive breast cancer cells to tamoxifen treatment. They report that the expression of c-ABL is a frequent event in primary breast cancer tumor tissues, and the expression of c-ABL in ER-positive tumors is significantly correlated with disease progression and metastasis. This work suggests $\mathrm{c}-\mathrm{ABL}$ as a potential therapeutic target and prognostic tumor marker for breast cancer. 


\section{CHAPTER 4. CONCLUSION}

Endocrine treatment of ER-positive breast cancer with tamoxifen, and later on with aromatase inhibitors and fulvestrant, was the first target-based therapeutic strategy in oncology [101]. Unfortunately, a substantial proportion of patients, despite being ER and/or PR positive, are either primarily resistant or will develop resistance during the course of their disease. Delineation of the molecular mechanisms underlying the development of resistance will allow development of treatment strategies to enhance response and compromise resistance.

It has become apparent that ER transcriptional actions are not just determined by the ligand, but also by complex interactions between co-regulatory molecules and multiple signaling pathways, which provide several potential mechanisms by which cancer cells may become estrogen-independent and tamoxifen-resistant [17]. Experimental models have demonstrated that the development of resistance is accompanied by the many mechanisms discussed. Clinical data further support several of these mechanisms. Further elucidation of the clinically relevant pathways of resistance, biomarkers reflecting the activity of these pathways and surrogate biomarkers predictive of response are therefore critical to determine which therapeutic strategies should be tested [102]. Some pharmacological agents targeting these pathways are currently clinically available and others are in development, but it is imperative to remember that there are likely to be several clinically significant pathways to resistance and that some will be important in certain patients but irrelevant in others. Gene expression analysis and molecular profiling have increased our understanding of the mechanisms of response and resistance, and these approaches are currently being used to develop gene signatures that may predict clinical responses to treatment [11].

Resistance to SERMs, aromatase inhibitors, and SERDs are likely to be mediated by distinct pathways, which explains the lack of cross-resistance between the different classes of drugs [15]. Therefore, the sequential use of endocrine agents is the supported treatment plan, but the currently available clinical, experimental, and pharmacokinetic data do not support the concomitant use of hormonal agents [15]. However, combinatorial therapies of tamoxifen (or other hormonal agents) with drugs aimed at the signaling pathways underlying the development of resistance may be a potential means of delaying the onset of resistance. A number of clinical trials have sought to determine whether the addition of signal transduction inhibitors to endocrine therapy may overcome endocrine resistance or delay its development [102]. Trials using HER2/neu antagonists, tyrosine kinase inhibitors, multikinase inhibitors, src inhibitors, and mTOR antagonists are currently underway in the treatment of breast cancer. Despite an improved understanding, resistance to all forms of endocrine therapy remains a significant clinical problem. A clearer understanding of the clinically significant mechanisms by which resistance develops is critical for improving the treatment of patients with breast cancer as well as in the development of new drugs and optimal treatment strategies. 


\section{LIST OF REFERENCES}

1. American Cancer Society. Breast Cancer Facts \& Figures 2009-2010. Atlanta: American Cancer Society, Inc.

2. GT., B., On the treatment of inoperable cases of carcinoma of the mamma: suggestion for a new method of treatment, with illustrative cases. Lancet, 1896. 2: p. 104-7.

3. Engelsman, E., et al., Human breast cancer and estrogen receptor. Arch Chir Neerl, 1973. 25(4): p. 393-7.

4. Yager, J.D. and N.E. Davidson, Estrogen carcinogenesis in breast cancer. N Engl J Med, 2006. 354(3): p. 270-82.

5. Lee, W.L., et al., The role of selective estrogen receptor modulators on breast cancer: from tamoxifen to raloxifene. Taiwan J Obstet Gynecol, 2008. 47(1): p. 24-31.

6. Breast cancer and hormone replacement therapy: collaborative reanalysis of data from 51 epidemiological studies of 52,705 women with breast cancer and 108,411 women without breast cancer. Collaborative Group on Hormonal Factors in Breast Cancer. Lancet, 1997. 350(9084): p. 1047-59.

7. Cheung, K.L., Endocrine therapy for breast cancer: an overview. Breast, 2007. 16(4): p. 327-43.

8. Allen, E. and E.A. Doisy, Landmark article Sept 8, 1923. An ovarian hormone. Preliminary report on its localization, extraction and partial purification, and action in test animals. By Edgar Allen and Edward A. Doisy. JAMA, 1983. 250(19): p. 2681-3.

9. Jensen, E.V. and V.C. Jordan, The estrogen receptor: a model for molecular medicine. Clin Cancer Res, 2003. 9(6): p. 1980-9.

10. Massarweh, S. and R. Schiff, Unraveling the mechanisms of endocrine resistance in breast cancer: new therapeutic opportunities. Clin Cancer Res, 2007. 13(7): $\mathrm{p}$. $1950-4$.

11. Riggins, R.B., et al., Pathways to tamoxifen resistance. Cancer Lett, 2007. 256(1): p. $1-24$.

12. Ascenzi, P., A. Bocedi, and M. Marino, Structure-function relationship of estrogen receptor alpha and beta: impact on human health. Mol Aspects Med, 2006. 27(4): p. 299-402. 
13. Deroo, B.J. and K.S. Korach, Estrogen receptors and human disease. J Clin Invest, 2006. 116(3): p. 561-70.

14. Dahlman-Wright, K., et al., International Union of Pharmacology. LXIV. Estrogen receptors. Pharmacol Rev, 2006. 58(4): p. 773-81.

15. Zilli, M., et al., Molecular mechanisms of endocrine resistance and their implication in the therapy of breast cancer. Biochim Biophys Acta, 2009. 1795(1): p. $62-81$.

16. Hall, J.M. and D.P. McDonnell, The estrogen receptor beta-isoform (ERbeta) of the human estrogen receptor modulates ERalpha transcriptional activity and is a key regulator of the cellular response to estrogens and antiestrogens. Endocrinology, 1999. 140(12): p. 5566-78.

17. Ring, A. and M. Dowsett, Mechanisms of tamoxifen resistance. Endocr Relat Cancer, 2004. 11(4): p. 643-58.

18. Frasor, J., et al., Profiling of estrogen up- and down-regulated gene expression in human breast cancer cells: insights into gene networks and pathways underlying estrogenic control of proliferation and cell phenotype. Endocrinology, 2003. 144(10): p. 4562-74.

19. Gruber, C.J., et al., Anatomy of the estrogen response element. Trends Endocrinol Metab, 2004. 15(2): p. 73-8.

20. Razandi, M., et al., Proximal events in signaling by plasma membrane estrogen receptors. J Biol Chem, 2003. 278(4): p. 2701-12.

21. Howell, S.J., S.R. Johnston, and A. Howell, The use of selective estrogen receptor modulators and selective estrogen receptor down-regulators in breast cancer. Best Pract Res Clin Endocrinol Metab, 2004. 18(1): p. 47-66.

22. Rugo, H.S., The breast cancer continuum in hormone-receptor-positive breast cancer in postmenopausal women: evolving management options focusing on aromatase inhibitors. Ann Oncol, 2008. 19(1): p. 16-27.

23. Nadji, M., et al., Immunohistochemistry of estrogen and progesterone receptors reconsidered: experience with 5,993 breast cancers. Am J Clin Pathol, 2005. 123(1): p. 21-7.

24. Riggs, B.L. and L.C. Hartmann, Selective estrogen-receptor modulators -mechanisms of action and application to clinical practice. N Engl J Med, 2003. 348(7): p. 618-29. 
25. Mokbel, K., The evolving role of aromatase inhibitors in breast cancer. Int J Clin Oncol, 2002. 7(5): p. 279-83.

26. Johnston, S.R., New Strategies in Estrogen Receptor-Positive Breast Cancer. Clin Cancer Res, 2010. 16(7): p. 1979-87.

27. Jordan, V.C., Tamoxifen: a most unlikely pioneering medicine. Nat Rev Drug Discov, 2003. 2(3): p. 205-13.

28. Jordan, V.C., Tamoxifen (ICI46,474) as a targeted therapy to treat and prevent breast cancer. Br J Pharmacol, 2006. 147 Suppl 1: p. S269-76.

29. Jordan, V.C., Fourteenth Gaddum Memorial Lecture. A current view of tamoxifen for the treatment and prevention of breast cancer. Br J Pharmacol, 1993. 110(2): p. 507-17.

30. Fisher, B., et al., Tamoxifen for prevention of breast cancer: report of the National Surgical Adjuvant Breast and Bowel Project P-1 Study. J Natl Cancer Inst, 1998. 90(18): p. 1371-88.

31. Patel, R.R., C.G. Sharma, and V.C. Jordan, Optimizing the antihormonal treatment and prevention of breast cancer. Breast Cancer, 2007. 14(2): p. 113-22.

32. Jordan, V.C. and B.W. O'Malley, Selective estrogen-receptor modulators and antihormonal resistance in breast cancer. J Clin Oncol, 2007. 25(36): p. 5815-24.

33. Lewis, J.S. and V.C. Jordan, Selective estrogen receptor modulators (SERMs): mechanisms of anticarcinogenesis and drug resistance. Mutat Res, 2005. 591(12): p. 247-63.

34. Shang, Y. and M. Brown, Molecular determinants for the tissue specificity of SERMs. Science, 2002. 295(5564): p. 2465-8.

35. Tamoxifen for early breast cancer: an overview of the randomised trials. Early Breast Cancer Trialists' Collaborative Group. Lancet, 1998. 351(9114): p. 145167.

36. Johnson, M.D., et al., Pharmacological characterization of 4-hydroxy-Ndesmethyl tamoxifen, a novel active metabolite of tamoxifen. Breast Cancer Res Treat, 2004. 85(2): p. 151-9.

37. Jordan, V.C., New insights into the metabolism of tamoxifen and its role in the treatment and prevention of breast cancer. Steroids, 2007. 72(13): p. 829-42.

38. Clarke, R., et al., Cellular and molecular pharmacology of antiestrogen action and resistance. Pharmacol Rev, 2001. 53(1): p. 25-71. 
39. Parl, F.F., Multiple mechanisms of estrogen receptor gene repression contribute to ER-negative breast cancer. Pharmacogenomics J, 2003. 3(5): p. 251-3.

40. Ottaviano, Y.L., et al., Methylation of the estrogen receptor gene $\mathrm{CpG}$ island marks loss of estrogen receptor expression in human breast cancer cells. Cancer Res, 1994. 54(10): p. 2552-5.

41. Gutierrez, M.C., et al., Molecular changes in tamoxifen-resistant breast cancer: relationship between estrogen receptor, HER-2, and p38 mitogen-activated protein kinase. J Clin Oncol, 2005. 23(11): p. 2469-76.

42. Herynk, M.H. and S.A. Fuqua, Estrogen receptor mutations in human disease. Endocr Rev, 2004. 25(6): p. 869-98.

43. Roodi, N., et al., Estrogen receptor gene analysis in estrogen receptor-positive and receptor-negative primary breast cancer. J Natl Cancer Inst, 1995. 87(6): p. 44651.

44. Pettersson, K., F. Delaunay, and J.A. Gustafsson, Estrogen receptor beta acts as a dominant regulator of estrogen signaling. Oncogene, 2000. 19(43): p. 4970-8.

45. Girault, I., I. Bieche, and R. Lidereau, Role of estrogen receptor alpha transcriptional coregulators in tamoxifen resistance in breast cancer. Maturitas, 2006. 54(4): p. 342-51.

46. Osborne, C.K., et al., Role of the estrogen receptor coactivator AIB1 (SRC-3) and HER-2/neu in tamoxifen resistance in breast cancer. J Natl Cancer Inst, 2003. 95(5): p. 353-61.

47. Anzick, S.L., et al., AIB1, a steroid receptor coactivator amplified in breast and ovarian cancer. Science, 1997. 277(5328): p. 965-8.

48. Webb, P., et al., Estrogen receptor activation function 1 works by binding p160 coactivator proteins. Mol Endocrinol, 1998. 12(10): p. 1605-18.

49. Lavinsky, R.M., et al., Diverse signaling pathways modulate nuclear receptor recruitment of N-CoR and SMRT complexes. Proc Natl Acad Sci U S A, 1998. 95(6): p. 2920-5.

50. Girault, I., et al., Expression analysis of estrogen receptor alpha coregulators in breast carcinoma: evidence that NCOR1 expression is predictive of the response to tamoxifen. Clin Cancer Res, 2003. 9(4): p. 1259-66.

51. Nicholson, R.I., et al., Growth factor signalling networks in breast cancer and resistance to endocrine agents: new therapeutic strategies. J Steroid Biochem Mol Biol, 2005. 93(2-5): p. 257-62. 
52. Nicholson, R.I., et al., Nonendocrine pathways and endocrine resistance: observations with antiestrogens and signal transduction inhibitors in combination. Clin Cancer Res, 2004. 10(1 Pt 2): p. 346S-54S.

53. Yarden, R.I., M.A. Wilson, and S.A. Chrysogelos, Estrogen suppression of EGFR expression in breast cancer cells: a possible mechanism to modulate growth. J Cell Biochem Suppl, 2001. Suppl 36: p. 232-46.

54. Gee, J.M., et al., The antiepidermal growth factor receptor agent gefitinib (ZD1839/Iressa) improves antihormone response and prevents development of resistance in breast cancer in vitro. Endocrinology, 2003. 144(11): p. 5105-17.

55. van Agthoven, T., et al., Ectopic expression of epidermal growth factor receptors induces hormone independence in ZR-75-1 human breast cancer cells. Cancer Res, 1992. 52(18): p. 5082-8.

56. Hutcheson, I.R., et al., Oestrogen receptor-mediated modulation of the EGFR/MAPK pathway in tamoxifen-resistant MCF-7 cells. Breast Cancer Res Treat, 2003. 81(1): p. 81-93.

57. Fan, P., et al., Long-term treatment with tamoxifen facilitates translocation of estrogen receptor alpha out of the nucleus and enhances its interaction with EGFR in MCF-7 breast cancer cells. Cancer Res, 2007. 67(3): p. 1352-60.

58. Knowlden, J.M., et al., Insulin-like growth factor-I receptor signaling in tamoxifen-resistant breast cancer: a supporting role to the epidermal growth factor receptor. Endocrinology, 2005. 146(11): p. 4609-18.

59. Massarweh, S., et al., Tamoxifen resistance in breast tumors is driven by growth factor receptor signaling with repression of classic estrogen receptor genomic function. Cancer Res, 2008. 68(3): p. 826-33.

60. Campbell, R.A., et al., Phosphatidylinositol 3-kinase/AKT-mediated activation of estrogen receptor alpha: a new model for anti-estrogen resistance. J Biol Chem, 2001. 276(13): p. 9817-24.

61. Parisot, J.P., et al., Altered expression of the IGF-1 receptor in a tamoxifenresistant human breast cancer cell line. Br J Cancer, 1999. 79(5-6): p. 693-700.

62. Cohen, B.D., et al., Combination therapy enhances the inhibition of tumor growth with the fully human anti-type 1 insulin-like growth factor receptor monoclonal antibody CP-751,871. Clin Cancer Res, 2005. 11(5): p. 2063-73.

63. Lu, Y., et al., Insulin-like growth factor-I receptor signaling and resistance to trastuzumab (Herceptin). J Natl Cancer Inst, 2001. 93(24): p. 1852-7. 
64. Nahta, R., et al., Insulin-like growth factor-I receptor/human epidermal growth factor receptor 2 heterodimerization contributes to trastuzumab resistance of breast cancer cells. Cancer Res, 2005. 65(23): p. 11118-28.

65. Haluska, P., et al., HER receptor signaling confers resistance to the insulin-like growth factor-I receptor inhibitor, BMS-536924. Mol Cancer Ther, 2008. 7(9): p. 2589-98.

66. Weroha, S.J. and P. Haluska, IGF-1 receptor inhibitors in clinical trials--early lessons. J Mammary Gland Biol Neoplasia, 2008. 13(4): p. 471-83.

67. van der Flier, S., et al., Bcar1/p130Cas protein and primary breast cancer: prognosis and response to tamoxifen treatment. J Natl Cancer Inst, 2000. 92(2): p. $120-7$.

68. Defilippi, P., P. Di Stefano, and S. Cabodi, p130Cas: a versatile scaffold in signaling networks. Trends Cell Biol, 2006. 16(5): p. 257-63.

69. Riggins, R.B., et al., Physical and functional interactions between Cas and c-Src induce tamoxifen resistance of breast cancer cells through pathways involving epidermal growth factor receptor and signal transducer and activator of transcription 5b. Cancer Res, 2006. 66(14): p. 7007-15.

70. Planas-Silva, M.D. and K.N. Hamilton, Targeting c-Src kinase enhances tamoxifen's inhibitory effect on cell growth by modulating expression of cell cycle and survival proteins. Cancer Chemother Pharmacol, 2007. 60(4): p. 53543.

71. van Agthoven, T., et al., Identification of BCAR3 by a random search for genes involved in antiestrogen resistance of human breast cancer cells. EMBO J, 1998. 17(10): p. 2799-808.

72. Felekkis, K.N., et al., AND-34 activates phosphatidylinositol 3-kinase and induces anti-estrogen resistance in a SH2 and GDP exchange factor-like domaindependent manner. Mol Cancer Res, 2005. 3(1): p. 32-41.

73. Faridi, J., et al., Expression of constitutively active Akt-3 in MCF-7 breast cancer cells reverses the estrogen and tamoxifen responsivity of these cells in vivo. Clin Cancer Res, 2003. 9(8): p. 2933-9.

74. Jordan, N.J., et al., Increased constitutive activity of PKB/Akt in tamoxifen resistant breast cancer MCF-7 cells. Breast Cancer Res Treat, 2004. 87(2): p. 16780. 
75. Likhite, V.S., et al., Kinase-specific phosphorylation of the estrogen receptor changes receptor interactions with ligand, deoxyribonucleic acid, and coregulators associated with alterations in estrogen and tamoxifen activity. Mol Endocrinol, 2006. 20(12): p. 3120-32.

76. Mamane, Y., et al., mTOR, translation initiation and cancer. Oncogene, 2006. 25(48): p. 6416-22.

77. deGraffenried, L.A., et al., Inhibition of mTOR activity restores tamoxifen response in breast cancer cells with aberrant Akt Activity. Clin Cancer Res, 2004. 10(23): p. 8059-67.

78. Angel, P. and M. Karin, The role of Jun, Fos and the AP-1 complex in cellproliferation and transformation. Biochim Biophys Acta, 1991. 1072(2-3): p. 12957.

79. Dumont, J.A., et al., Progression of MCF-7 breast cancer cells to antiestrogenresistant phenotype is accompanied by elevated levels of AP-1 DNA-binding activity. Cell Growth Differ, 1996. 7(3): p. 351-9.

80. Johnston, S.R., et al., Increased activator protein-1 DNA binding and c-Jun NH2terminal kinase activity in human breast tumors with acquired tamoxifen resistance. Clin Cancer Res, 1999. 5(2): p. 251-6.

81. Schiff, R., et al., Oxidative stress and AP-1 activity in tamoxifen-resistant breast tumors in vivo. J Natl Cancer Inst, 2000. 92(23): p. 1926-34.

82. Nair, B.C. and R.K. Vadlamudi, Regulation of hormonal therapy resistance by cell cycle machinery. Gene Ther Mol Biol, 2008. 12: p. 395.

83. Butt, A.J., et al., Downstream targets of growth factor and oestrogen signalling and endocrine resistance: the potential roles of c-Myc, cyclin D1 and cyclin E. Endocr Relat Cancer, 2005. 12 Suppl 1: p. S47-59.

84. Kilker, R.L., et al., Cyclin D1 expression is dependent on estrogen receptor function in tamoxifen-resistant breast cancer cells. J Steroid Biochem Mol Biol, 2004. 92(1-2): p. 63-71.

85. Kilker, R.L. and M.D. Planas-Silva, Cyclin D1 is necessary for tamoxifeninduced cell cycle progression in human breast cancer cells. Cancer Res, 2006. 66(23): p. 11478-84.

86. Zwijsen, R.M., et al., CDK-independent activation of estrogen receptor by cyclin D1. Cell, 1997. 88(3): p. 405-15. 
87. Rudas, M., et al., Cyclin D1 expression in breast cancer patients receiving adjuvant tamoxifen-based therapy. Clin Cancer Res, 2008. 14(6): p. 1767-74.

88. Stendahl, M., et al., Cyclin D1 overexpression is a negative predictive factor for tamoxifen response in postmenopausal breast cancer patients. Br J Cancer, 2004. 90(10): p. 1942-8.

89. Jirstrom, K., et al., Adverse effect of adjuvant tamoxifen in premenopausal breast cancer with cyclin D1 gene amplification. Cancer Res, 2005. 65(17): p. 8009-16.

90. Caldon, C.E., et al., Cell cycle control in breast cancer cells. J Cell Biochem, 2006. 97(2): p. 261-74.

91. Dhillon, N.K. and M. Mudryj, Ectopic expression of cyclin E in estrogen responsive cells abrogates antiestrogen mediated growth arrest. Oncogene, 2002. 21(30): p. 4626-34.

92. Cariou, S., et al., Down-regulation of p21WAF1/CIP1 or p27Kip1 abrogates antiestrogen-mediated cell cycle arrest in human breast cancer cells. Proc Natl Acad Sci U S A, 2000. 97(16): p. 9042-6.

93. Carroll, J.S., et al., p27(Kip1) induces quiescence and growth factor insensitivity in tamoxifen-treated breast cancer cells. Cancer Res, 2003. 63(15): p. 4322-6.

94. Abukhdeir, A.M., et al., Tamoxifen-stimulated growth of breast cancer due to $\mathrm{p} 21$ loss. Proc Natl Acad Sci U S A, 2008. 105(1): p. 288-93.

95. Prall, O.W., et al., c-Myc or cyclin D1 mimics estrogen effects on cyclin E-Cdk2 activation and cell cycle reentry. Mol Cell Biol, 1998. 18(8): p. 4499-508.

96. Mukherjee, S. and S.E. Conrad, c-Myc suppresses p21WAF1/CIP1 expression during estrogen signaling and antiestrogen resistance in human breast cancer cells. J Biol Chem, 2005. 280(18): p. 17617-25.

97. Pohl, G., et al., High p27Kip1 expression predicts superior relapse-free and overall survival for premenopausal women with early-stage breast cancer receiving adjuvant treatment with tamoxifen plus goserelin. J Clin Oncol, 2003. 21(19): p. 3594-600.

98. Perez-Tenorio, G., et al., Cytoplasmic p21WAF1/CIP1 correlates with Akt activation and poor response to tamoxifen in breast cancer. Int J Oncol, 2006. 28(5): p. 1031-42.

99. Pendergast, A.M., The Abl family kinases: mechanisms of regulation and signaling. Adv Cancer Res, 2002. 85: p. 51-100. 
100. Zhao, H., et al., Enhanced resistance to tamoxifen by the c-ABL proto-oncogene in breast cancer. Neoplasia, 2010. 12(3): p. 214-23.

101. Normanno, N., et al., Target-based therapies in breast cancer: current status and future perspectives. Endocr Relat Cancer, 2009. 16(3): p. 675-702.

102. Bedard, P.L., et al., Overcoming endocrine resistance in breast cancer: are signal transduction inhibitors the answer? Breast Cancer Res Treat, 2008. 108(3): p. 307-17. 


\section{VITA}

Cyrus McCoy Adams was born on February 24, 1985 in Indianapolis, IN. He graduated from Franklin and Marshall College in 2007 with a Bachelor of Arts. He worked for two years at Vanderbilt University Medical Center in a basic science research laboratory in the Urologic Surgery department. In 2009, he was accepted into the University of Tennessee Health Science Center Master's in Pharmacology degree program. Upon graduation in May of 2010, Cyrus will enroll in medical school. 